\title{
NMR structures and functional roles of two related chitin-binding domains of a lytic polysaccharide monooxygenase from Cellvibrio japonicus
}

Eva Madland*1, Zarah Forsberg*2, Yong Wang ${ }^{3}$, Kresten Lindorff-Larsen ${ }^{3}$, Axel Niebisch ${ }^{4}$, Jan Modregger $^{4}$, Vincent G. H. Eijsink ${ }^{2}$, Finn L. Aachmann ${ }^{1}$, Gaston Courtade ${ }^{1 * *}$

${ }^{1}$ Norwegian Biopolymer Laboratory (NOBIPOL), Department of Biotechnology and Food Science, NTNU Norwegian University of Science and Technology, 7491Trondheim, Norway

${ }^{2}$ Faculty of Chemistry, Biotechnology and Food Science, Norwegian University of Life Sciences (NMBU), 1432 Ås, Norway

${ }^{3}$ Structural Biology and NMR Laboratory, Linderstrøm-Lang Centre for Protein Science, Department of Biology, University of Copenhagen, Denmark

${ }^{4}$ Eucodis Bioscience GmbH, Campus Vienna Biocenter 2, 1030 Wien, Austria

*These authors contributed equally to this work

**Corresponding author

E-mail: gaston.courtade@ntnu.no

Keywords: carbohydrate-binding domain, chitin, lytic polysaccharide monooxygenase, molecular dynamics simulations, multi-modular, nuclear magnetic resonance (NMR), substrate binding 


\begin{abstract}
Among the extensive repertoire of carbohydrate-active enzymes, lytic polysaccharide monooxygenases (LPMOs) have a key role in recalcitrant biomass degradation. LPMOs are copper-dependent enzymes that catalyze oxidative cleavage of glycosidic bonds in polysaccharides such as cellulose and chitin. Several LPMOs contain carbohydrate-binding modules (CBMs) that are known to promote LPMO efficiency. Still, structural and functional properties of some of these CBMs remain unknown and it is not clear why some LPMOs, like CjLPMO10A from Cellvibrio japonicus, have two CBMs (CjCBM5 and CjCBM73). Here, we studied substrate binding by these two CBMs to shine light on the functional variation, and determined the solution structures of both by NMR, which includes the first structure of a member of the CBM73 family. Chitin-binding experiments and molecular dynamics simulations showed that, while both CBMs bind crystalline chitin with $K_{\mathrm{d}}$ values in the $\mu \mathrm{M}$ range, $C j \mathrm{CBM} 73$ has higher affinity than $C j$ CBM5. Furthermore, NMR titration experiments showed that $C j C B M 5$ binds soluble chitohexaose, whereas no binding to soluble chitin was detected for CjCBM73. These functional differences correlated with distinctly different architectures of the substrate-binding surfaces of the two CBMs. Taken together, these results provide insight into natural variation among related chitin-binding CBMs and the possible functional implications of such variation.
\end{abstract}

\title{
Introduction
}

Chitin is a linear, water insoluble polysaccharide composed of $\beta$-1,4-linked $N$-acetyl-Dglucosamine (GlcNAc) units found in the cell wall matrix of fungi and the exoskeletons of arthropods. Despite being the second most abundant polymer in nature, after cellulose, chitin does not accumulate in most ecosystems and tends to be absent in fossils (1). This is testimony to the capacity of nature to depolymerize and recycle chitin.

Chitinases (EC 3.2.1.14) catalyze the hydrolytic degradation of chitin and belong to the glycoside hydrolase $(\mathrm{GH})$ class of carbohydrate-active enzymes. Even though GHs efficiently degrade amorphous regions of chitin (2-4), they are inefficient at degrading crystalline chitin (5). The discovery of lytic polysaccharide monooxygenases (LPMOs) $(6,7)$ has given new insights into the degradation of chitin and other structural polysaccharides. LPMOs are copper-dependent enzymes that catalyze oxidative cleavage of glycosidic bonds in crystalline polysaccharides $(6,8)$. Aside from chitin, LPMOs have been reported to act on polysaccharides such as cellulose (8-11), various hemicelluloses (12) and starch (13). In the degradation of chitin, LPMOs act in synergy with chitinases $(4,7)$. It is thought that LPMOs oxidize crystalline surfaces, causing "nicks" that lead to reduced crystallinity and introduction of new access points for chitinases $(6,10,14)$.

Carbohydrate Active enZymes (CAZymes), such as chitinases and LPMOs, may just be composed of a single catalytic domain, or may contain one or more non-catalytic domains such as carbohydrate-binding modules (CBMs). Currently, the CAZy database (15) contains 88 families of CBMs with a wide variety of binding specificities, including crystalline polysaccharides and short, soluble oligosaccharides $(16,17)$. The major role of CBMs is to keep an enzyme in close proximity of a substrate, thereby enhancing the effective concentration of the enzyme and overall reaction efficiency (16). In the context of LPMOs, CBMs may have a particularly important role because proximity to the substrate not only contributes to enzyme efficiency, but also protects the enzyme from autocatalytic inactivation. Several studies have shown that removal of CBMs has a 
negative effect on LPMO performance (18-22). There are multiple families of chitin-binding and cellulose-binding CBMs, which may have different binding specificities (e.g. $(18,23)$ ). For example, it has been shown that two cellulose-binding modules belonging to two different CBM families bind to different parts of cellulose (23). It is not trivial to predict or determine the role of CBMs and a better understanding of the ways in which they bind their substrates is needed.

To address functional variation among chitin-binding CBMs, we have used chitin-active CjLPMO10A from Cellvibro japonicus as a model system. The catalytic domain of this LPMO, which belongs to the auxiliary activity family 10 (AA10) in CAZy, is appended to two chitinbinding CBMs, an internal family 5 CBM (CjCBM5) and C-terminal family $73 \mathrm{CBM}(C j \mathrm{CBM} 73)$ (Figure 1). The three domains of $C j L P M O 10 A$ are connected by linkers that are rich in serine residues and are both approximately 30 amino acids long (Figure 1). A previous study has shown that both CBMs bind to $\alpha$ - and $\beta$-chitin, thus enhancing substrate-binding by the LPMO, and that the full length protein is more efficient in comparison to the catalytic domain alone (20). In the present study, we have compared multiple truncated variants of $C j$ LPMO10A (see Figure 1A) to understand the roles of the appended CBMs in LPMO functionality. Furthermore, we have used NMR spectroscopy to elucidate the solution structures of the two CBMs, CjCBM5 and CjCBM73, where the latter is the first structure to be determined for a member of the CBM73 family. We also used NMR titration experiments to investigate binding of the CBMs to chitohexaose. These results were complemented with molecular dynamics simulations to gain more insights into CBM binding to $\alpha$-chitin. Overall, the results show that while $C j \mathrm{CBM} 5$ and $C j \mathrm{CBM} 73$ are similar in overall structure and both bind to crystalline chitin, they differ in apparent melting temperature, binding site architecture and the ability to bind individual chitin chains. 


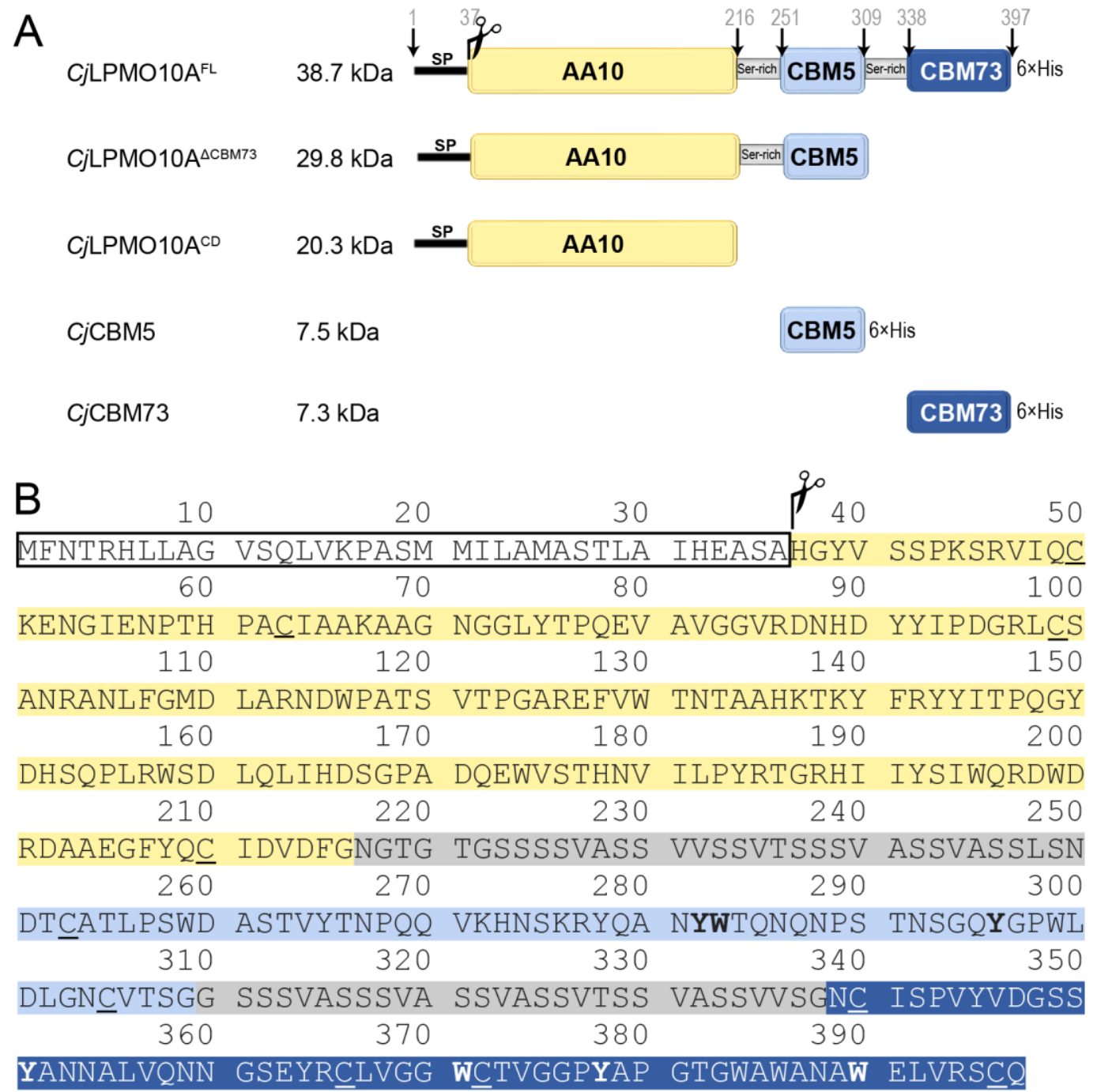

Figure 1. (A) Domain architecture and molecular weight of $C j L P M O 10 A$ and the truncated variants used in this study. The numbers above the full-length enzyme show the transitions between the domains and the linkers. The signal peptide (residue 1-37) is cleaved off during secretion. The indicated molecular weights are based on the mature protein, i.e. signal peptide free enzymes. Abbreviations used: SP, signal peptide; FL, full-length; CD, catalytic domain; Ser-rich linker; His $\times 6$, poly-histidine tag. (B) Primary sequence of $C j \mathrm{LPMO} 10 \mathrm{~A}^{\mathrm{FL}}$ with color coding according to panel A. Aromatic residues located on the binding surfaces of the two CBMs, as determined in this study, are printed in bold face; cysteine residues involved in disulfide bonds are underlined. 


\section{Results}

The effect of CBMs on chitin oxidation is substrate concentration dependent

To better understand the functional roles of CjCBM5 and CjCBM73 in relation to full-length $C j$ LPMO10A we started by testing the performance of the three catalytically active versions of $C j \mathrm{LPMO} 10 \mathrm{~A}, C j \mathrm{LPMO} 10 \mathrm{~A}^{\mathrm{FL}}$ (FL for full-length), $C j \mathrm{LPMO} 10 \mathrm{~A}^{\Delta \mathrm{CBM}^{2} 3}$ (for truncation of the CBM73 domain; see Figure 1A) and fully truncated $C j$ LPMO10A $^{\mathrm{CD}}$ (CD for catalytic domain) at different concentrations of $\alpha$-chitin (2, 10 or $50 \mathrm{~g} / \mathrm{L}$, see Figure 2). At all substrate concentrations, the full-length enzyme and the enzyme lacking only one CBM, CjLPMO10A ${ }^{\triangle \mathrm{CBM} 73}$, had similar progress curves and stayed active for the full duration of the experiment. At the two lowest substrate concentrations product formation by $C j L P M O 10 \mathrm{~A}^{\mathrm{CD}}$ ceased rapidly, and faster at the lowest substrate concentration, indicative of enzyme inactivation. However, at a substrate concentration of $50 \mathrm{~g} / \mathrm{L}$ all three variants showed similar progress curves and final product levels.

At the two lowest substrate concentrations, the amount of soluble oxidized products (relative to the total amount) was higher for the CBM containing variants of $C j \mathrm{LPMO} 10 \mathrm{~A}$ (> $85 \%$ ) compared to $C j \mathrm{LPMO} 10 \mathrm{~A}^{\mathrm{CD}}$ (about 50\%) (Figure 2D-E). This indicates that, at these lower substrate concentrations, the presence of at least one CBM leads to more localized oxidation, generating a higher fraction of short soluble products, as discussed in (22) and below. At the highest substrate concentration (Figure 2F), however, the fraction of soluble oxidized products was close to $50 \%$ for all three enzyme versions. All in all, the experiments depicted in Figure 2 did not show significant differences between the catalytic behavior of the two CBM containing variants, whereas deletion of both CBMs had a major effect.
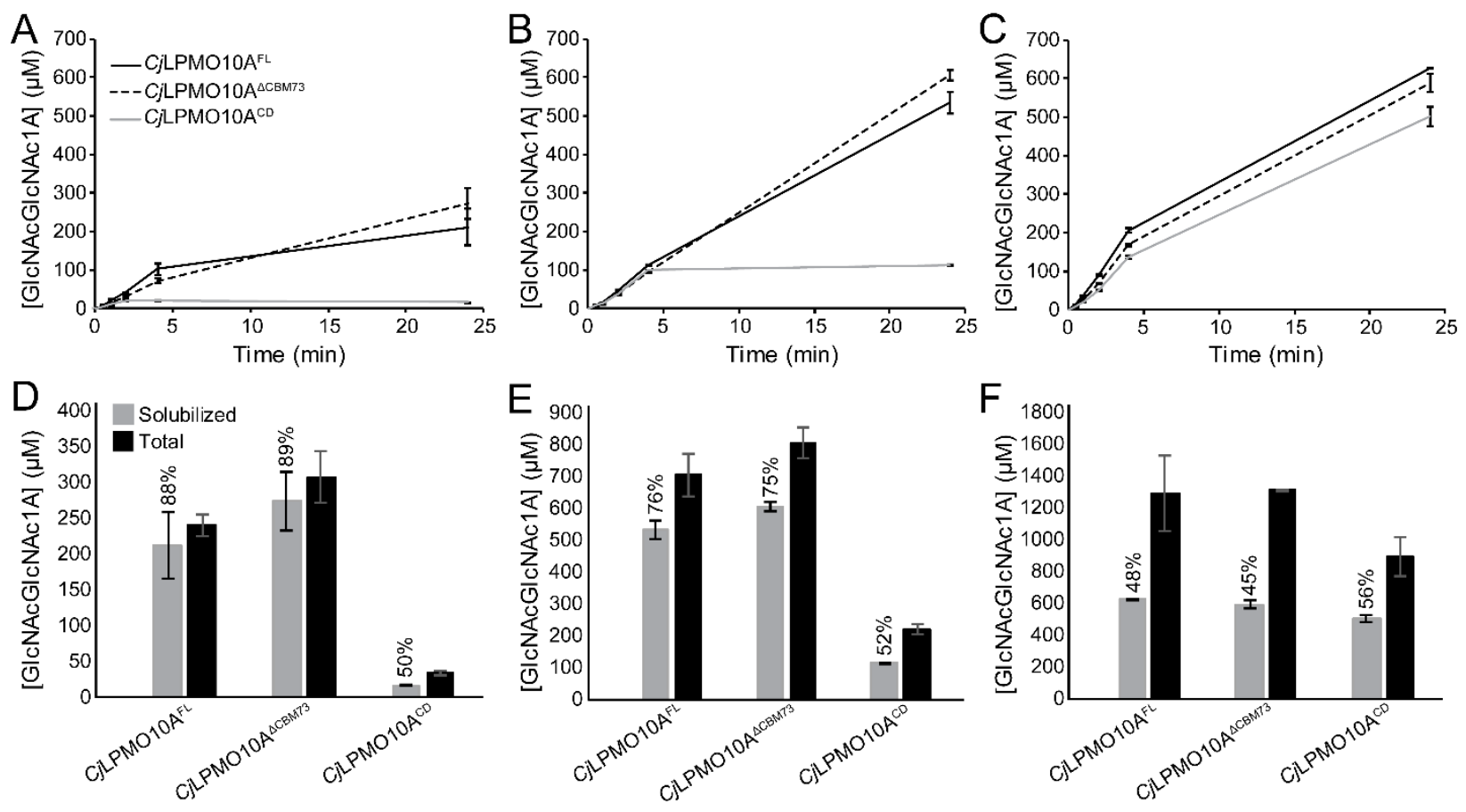

Figure 2. Chitin degradation by $C j \mathrm{LPMO} 10 \mathrm{~A}$ variants. Panels $\mathrm{A}-\mathrm{C}$ show progress curves for the formation of soluble oxidized products by $C j \mathrm{LPMO} \mathrm{AA}^{\mathrm{FL}}$ (solid black line), $C j \mathrm{LPMO} 10 \mathrm{~A}^{\triangle \mathrm{CBM} 73}$ (dashed black line) and $C j \mathrm{LPMO} 10 \mathrm{~A}^{\mathrm{CD}}$ (solid grey line) at substrate concentrations of $2 \mathrm{~g} / \mathrm{L}$ (A), $10 \mathrm{~g} / \mathrm{L}(\mathrm{B})$ and $50 \mathrm{~g} / \mathrm{L}(\mathrm{C}) \alpha$-chitin. Panel D - F show quantification of solubilized (grey bars) and 
total oxidized sites (black bars) after $24 \mathrm{~h}$ of LPMO incubation at the various substrate concentrations, i.e. $2 \mathrm{~g} / \mathrm{L}$ (D), $10 \mathrm{~g} / \mathrm{L}$ (E) and $50 \mathrm{~g} / \mathrm{L}$ (F). The fraction of soluble oxidized products is given as a percentage of the total for each reaction. All reactions were carried out with $0.5 \mu \mathrm{M}$ LPMO and $1 \mathrm{mM}$ ascorbic acid in $50 \mathrm{mM}$ sodium phosphate $\mathrm{pH} 7.0$ in a thermomixer set to $37^{\circ} \mathrm{C}$ and $800 \mathrm{rpm}$. For quantification of soluble products, the solubilized fraction was further degraded by $0.5 \mu \mathrm{M} S m \mathrm{CHB}$ prior to HPLC quantification. For quantification of total products (i.e. soluble and insoluble fraction), samples were heat inactivated after which all $\alpha$-chitin (diluted to $2 \mathrm{~g} / \mathrm{L}$ ) was degraded with a combination of $2.0 \mu \mathrm{M}$ SmChiA and $0.5 \mu \mathrm{M}$ SmCHB. The error bars show \pm s.d. $(n=3)$.

\section{Thermal stability and oxidative performance}

To assess possible functional differences between the full-length enzyme and the variant lacking only the CBM73, we analyzed the effect of temperature on the oxidative performance of these variants (Figure 3). It is believed that CAZymes with multiple CBMs have an advantage at elevated temperatures as the $\mathrm{CBM}(\mathrm{s})$ can counteract the loss of binding due to increased temperature (2426). Interestingly at the highest tested temperature $\left(70{ }^{\circ} \mathrm{C}\right) C_{\mathrm{LPMO}} \mathrm{LPA}^{\mathrm{FL}}$ showed significantly higher activity than $C j \mathrm{LPMO} 10 \mathrm{~A}^{\Delta \mathrm{CBM} 73}$. Thus, the presence of the CBM73 indeed has a beneficial effect on LPMO performance at higher temperatures. Determination of melting curves showed that the deletion of the $C j \mathrm{CBM} 73$ had some effect on the shape of the curve but not on the apparent melting temperature of approximately $70{ }^{\circ} \mathrm{C}$ (Figure S1). The apparent melting temperatures of the isolated CBMs were $57.2^{\circ} \mathrm{C}$ for $\mathrm{CjCBM} 5$ and $75.4{ }^{\circ} \mathrm{C}$ for $\mathrm{CjCBM} 73$, whereas the apparent melting temperature of the $C j \mathrm{LPMO} 10 \mathrm{~A}^{\mathrm{CD}}$ was $70.2^{\circ} \mathrm{C}$, which was reduced to $56.6^{\circ} \mathrm{C}$ upon removal of the copper.

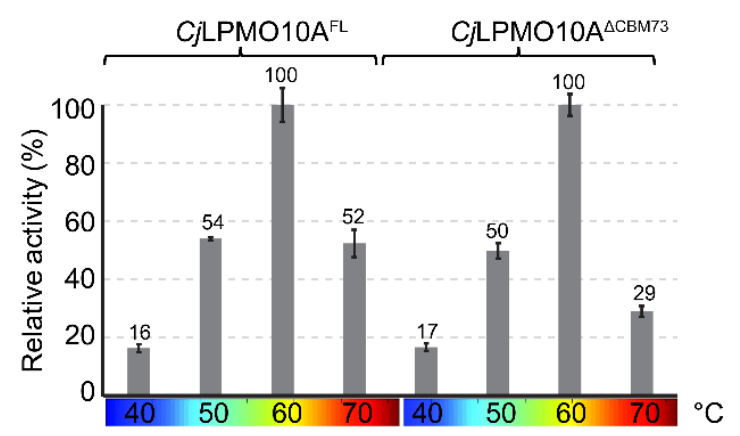

Figure 3. Catalytic performance of $C j \mathrm{LPMO} 10 \mathrm{~A}^{\mathrm{FL}}$ and $C j \mathrm{LPMO} 10 \mathrm{~A}^{\triangle \mathrm{CBM} 73}$ at varying temperatures. The relative activity was determined from linear progress curves for a 30-minute reaction. The $100 \%$ value corresponds to 61 and $47 \mu \mathrm{M}$ GlcNAcGlcNAc1A for $C j L P M O 10 A^{\mathrm{FL}}$ and $C j \mathrm{LPMO} 10 \mathrm{~A}^{\triangle \mathrm{CBM} 73}$, respectively. All reactions were carried out with $0.5 \mu \mathrm{M}$ LPMO, $10 \mathrm{~g} / \mathrm{L}$ $\alpha$-chitin and $1 \mathrm{mM}$ ascorbic acid in $50 \mathrm{mM}$ sodium phosphate $\mathrm{pH} 7.0$ in a thermomixer set to the indicated temperature and $800 \mathrm{rpm}$. Prior to product quantification the solubilized fraction was further degraded with $0.5 \mu \mathrm{M} S m \mathrm{CHB}$. Each point represents the average of values obtained in three independent experiments. 


\section{Solution structures of CjCBM5 and CjCBM73}

The solution structures of CjCBM5 (PDB: 6Z40) and CjCBM73 (PDB: 6Z41) were solved by NMR spectroscopy (Figure 4, Table S1). The chemical shift assignment completion for the backbone (N, $\mathrm{H}^{\mathrm{N}}, \mathrm{C}^{\alpha}, \mathrm{H}^{\alpha}$ and $\left.\mathrm{C}^{\prime}\right)$ and side chains (H and C) of CjCBM5 (BMRB: 34519). was > $88 \%$ and $>65 \%$, respectively, whereas these values were $>87 \%$ and $>59 \%$ for $C j \mathrm{CBM} 73$ (BMRB: 34520). Due to the cloning procedure, both proteins contained a Met at the N-terminus and an Ala followed by a 6xHis-tag at the C-terminus. For CjCBM5, no resonances from these additional amino acids were assigned, whereas for $C j \mathrm{CBM} 73$ the backbone resonances of the additional Ala and the first His in the 6xHis-tag were assigned.

A

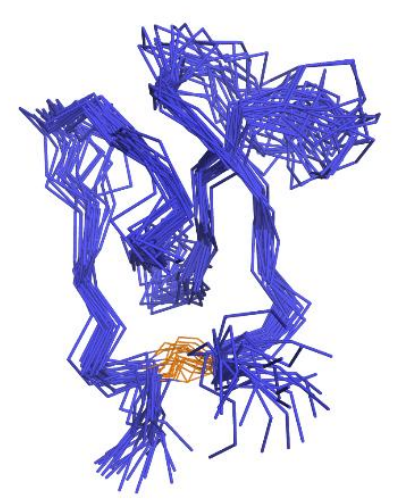

B

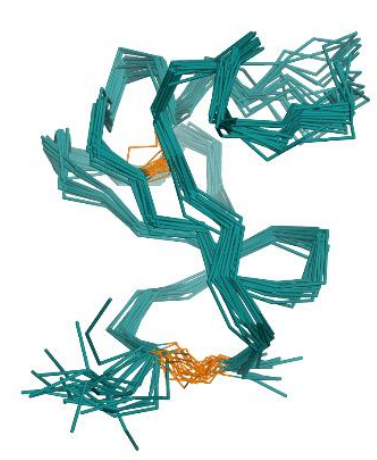

CjCBM5

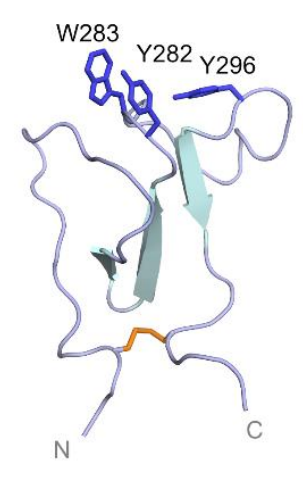

CjCBM73

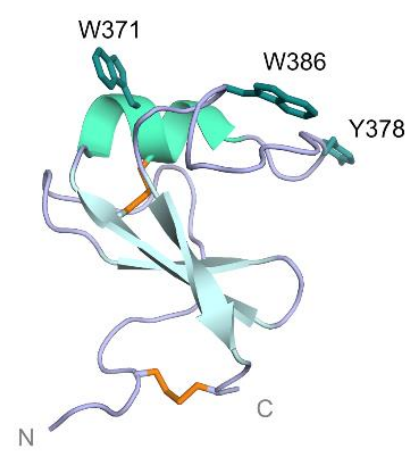

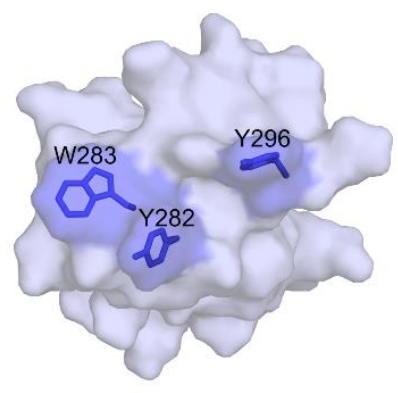

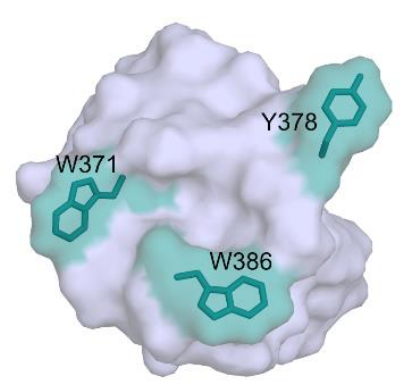

Figure 4. NMR solution structures of (A) CjCBM5 (PDB: 6Z40) and (B) CjCBM73 (PDB: 6Z41). The Figures show backbone representations for the 20 conformers with the lowest CYANA target function (left), a cartoon representation of the structure with the lowest target function (center), and a view of the binding surfaces (right). The cartoon representations also display the secondary structure elements as well as aromatic residues of the putative binding surface. Disulfide bridges (residues 253-306 in CjCBM5 and 340-396 and 366-372 for CjCBM73) are highlighted in orange. His-tags added for cloning purposes (see Experimental procedures) are not shown. 
The structures of CjCBM5 and CjCBM73 are similar $\left(\mathrm{C}^{\alpha}\right.$ rmsd $\left.5.6 \AA\right)$ and share the same overall fold (Figure 4). This fold has previously been described (27) as an "L" shape or "ski boot" fold due to the loop region attached perpendicularly to an anti-parallel $\beta$-sheet. The structures of both CBMs are stabilized by a disulfide bridge connecting the $\mathrm{N}$ - and $\mathrm{C}$-terminal ends of the domain. The structure of $C j \mathrm{CBM} 73$ shows a short $3{ }_{10}$ helix (residues $371-374$ ) that is linked to the central $\beta$-strand by an additional disulfide bridge. These features are unique for the CBM73 family (see Figure S2) and lack in CjCBM5 and other structurally characterized members of the CBM5 family.

Most CBMs rely on exposed aromatic residues that bind carbohydrates through $\mathrm{CH}-\pi$ interactions $(16,28)$. Based on structural information alone, Figure 4 shows that Y282, W283 and Y296 in CjCBM5, and W371, Y378 and W386 in CjCBM73 could be involved in substrate-binding. As shown in Figure S2, the aromatic pair Y282-W283 is almost fully conserved within the CBM5 family, whereas Y296 is less conserved. In the context of the CBM73 family (Figure S2), W371, Y378 and W386 appear to be highly conserved. To test interactions between chitin and these aromatic patches and their neighboring polar residues we performed NMR titrations with a soluble chitin substrate, chitohexaose, (GlcNAc) 6 .

\section{Probing interactions between soluble chitin and CBMs by NMR}

In the case of $C j \mathrm{CBM} 5$, titration with $(\mathrm{GlcNAc})_{6}$ led to significant chemical shift perturbation for W283 and Y296 as well as for residues in the neighboring loop region (T284, Q285 and G297) that are part of the putative binding surface (Figure 5). The chemical shift perturbations were used to calculate a $K_{\mathrm{d}}=2 \pm 1 \mathrm{mM}$. Of note this $K_{\mathrm{d}}$ value is some three orders of magnitude higher than the value obtained with solid $\alpha$-chitin (see below).

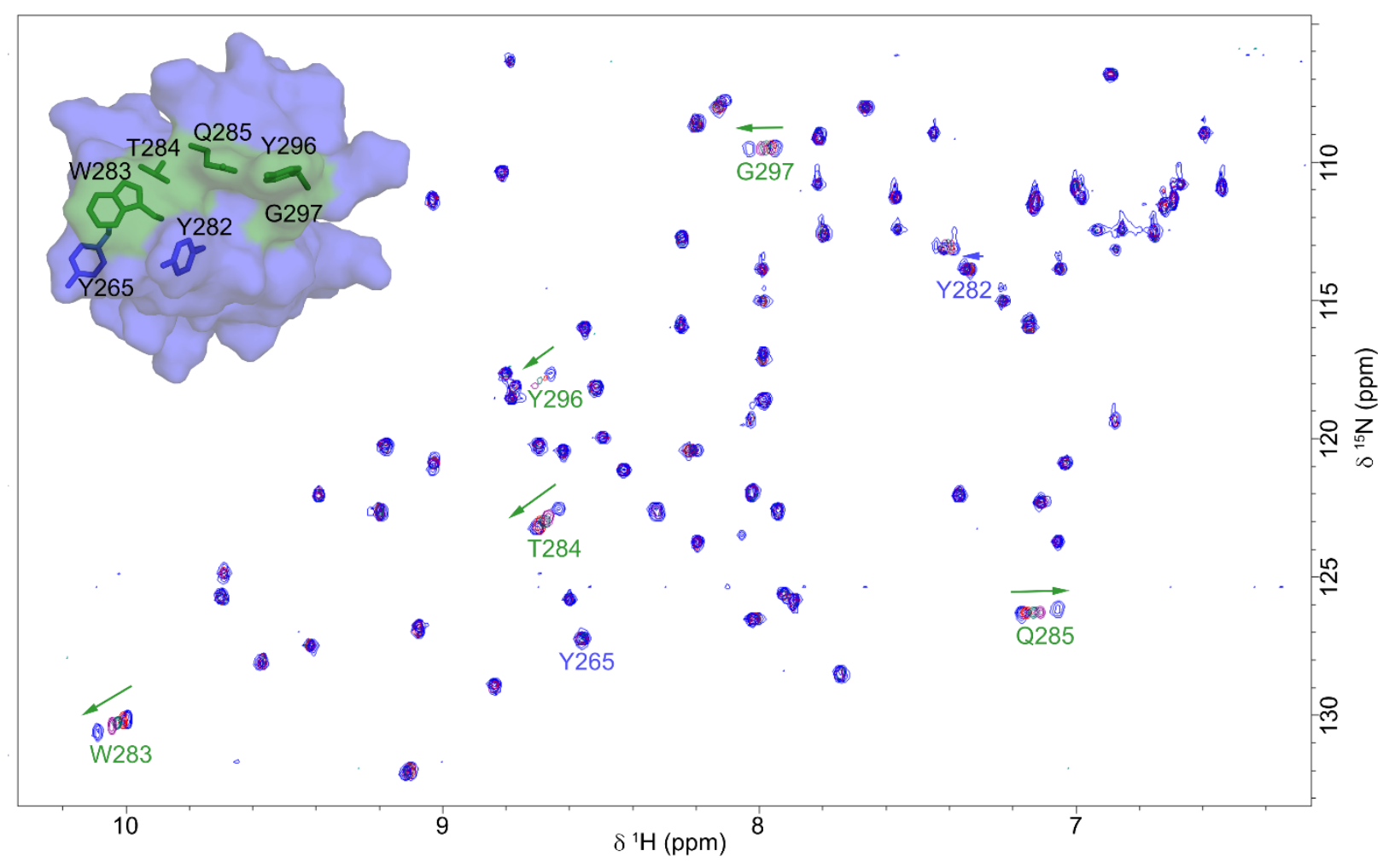


Figure 5. ${ }^{15} \mathrm{~N}-\mathrm{HSQC}$ of $\mathrm{CjCBM} 5$ interacting with $(\mathrm{GlcNAc})_{6}$. The picture shows an overlay of ${ }^{15} \mathrm{~N}$-HSQC spectra for $C j \mathrm{CBM} 5$ in the presence of $(\mathrm{GlcNAc})_{6}$ at various concentrations $(0.2,1.0$, 2.5 and $10 \mathrm{mM})$. The arrows indicate the direction of change in chemical shift perturbation as a result of the titration of CjCBM5with (GlcNAc) 6 . Affected residues (W283, T284, Q285, Y296 and G297) are highlighted in green on the surface model of CjCBM5. Other surface-exposed aromatic residues for which no significant chemical shift perturbation was detected (Y265 and Y282) are shown in blue for illustration purposes.

In stark contrast to the experiment with $C j \mathrm{CBM} 5$, titration of $C j \mathrm{CBM} 73$ with (GlcNAc) ${ }_{6}$ did not result in any significant chemical shift perturbations, indicating that this CBM does not bind this soluble substrate.

\section{Binding of CjCBM5 and CjCBM73 to oxidized and non-oxidized $\alpha$-chitin}

Previous binding studies have shown that both CBMs bind with micromolar affinity to both $\alpha$ - and $\beta$-chitin (20). These previous studies indicated similar $K_{\mathrm{d}}$ values (for $\alpha$-chitin) for $C j \mathrm{CBM} 5$ and CjCBM73. Here we tested binding using a similar setup, using both the same batch of $\alpha$-chitin and a batch of $\alpha$-chitin that had been pre-oxidized with $C j$ LPMO $10 \mathrm{~A}^{\mathrm{CD}}$ as described below and in Experimental procedures.

Oxidized chitin was prepared to assess whether surface oxidation would affect CBM binding, one idea being that gradual oxidation of the substrate surface could facilitate release of otherwise strongly bound CBMs. The material was prepared by treating chitin with the catalytic domain of $C j \mathrm{LPMO}_{10 \mathrm{~A}}^{\mathrm{CD}}$, followed by washing to remove solubilized oxidized chito-oligosaccharides and residual LPMO (see Experimental procedures for further details). The degree of oxidation of the solid fraction was determined upon complete enzymatic hydrolysis of the fraction, which entails that all oxidized sites end up as chitobionic acid. Data from six independent reactions, containing $20 \mathrm{mg} / \mathrm{mL}$ chitin, which corresponds to approximately $45 \mathrm{mM}$ of oxidized dimer in a theoretical $100 \%$ conversion reaction, indicated a degree of oxidation of about $0.3 \%$ (number obtained by dividing the chitobionic acid recovered from the solid fraction by the amount of chitobionic acid that would be obtained in a $100 \%$ conversion reaction). In an alternative approach, we divided the amount of chitobionic acid recovered from the solid fraction by the total amount of sugars (GlcNAc and chitobionic acid) recovered from this fraction, which indicated approximately $1 \%$ oxidation. Hence, the degree of oxidation of the insoluble fraction was estimated to be between $0.3 \%$ and $1 \%$ and we assume that oxidation essentially happened on the substrate surface.

Figure 6 shows binding curves for the two CBMs with "non-oxidized" (panel A and C) or "preoxidized" (panel B and D) $\alpha$-chitin. The data show that $C j \mathrm{CBM} 73\left(K_{\mathrm{d}}=2.9 \mu \mathrm{M}\right)$ binds with slightly higher affinity than $C j$ CBM5 $\left(K_{\mathrm{d}}=8.5 \mu \mathrm{M}\right)$. The binding studies with partly oxidized chitin showed similar results. The data showed a $\sim 20 \%$ increase in the $K_{\mathrm{d}}$ for $C j \mathrm{CBM} 5$, indicating that binding by this CBM may be negatively affected by surface oxidation, however, the difference was not statistically significant. 

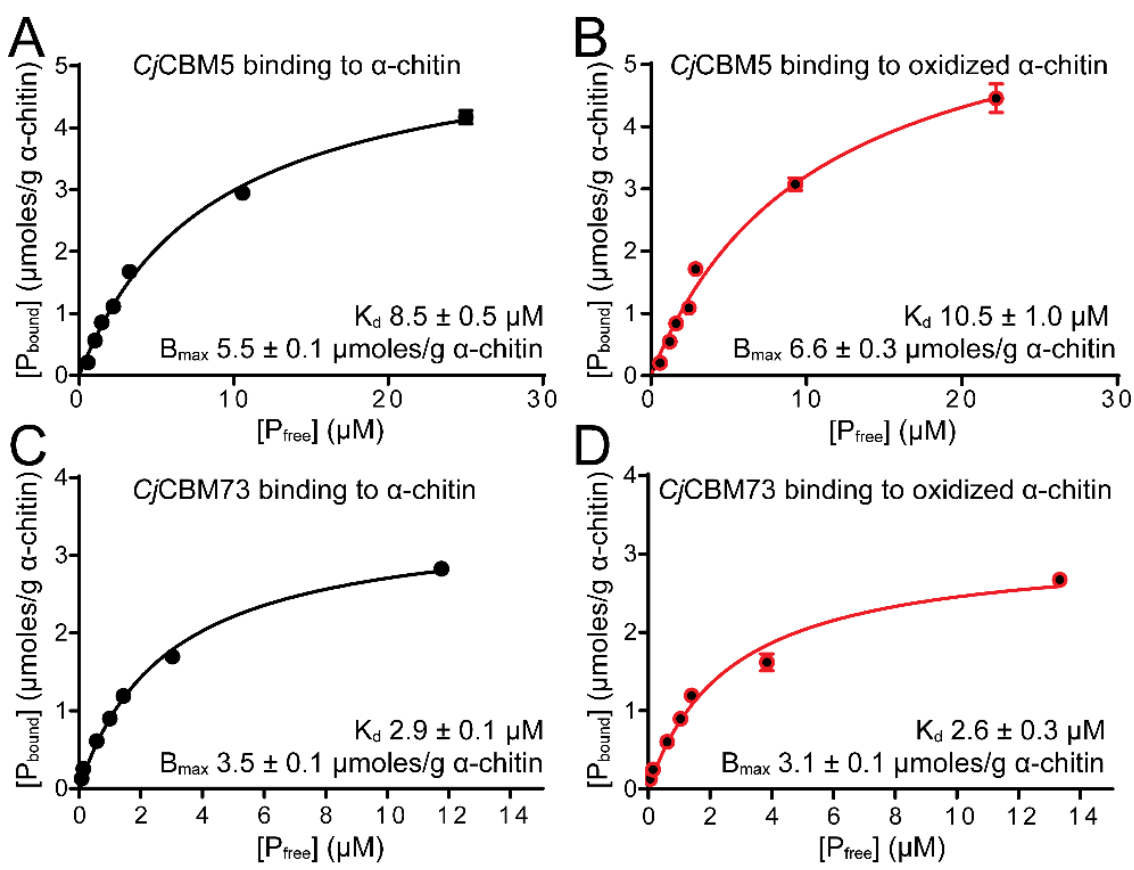

Figure 6. Binding of the CBMs of $C j \mathrm{LPMO} 10 \mathrm{~A}$ to $\alpha$-chitin. The plots show binding data for CjCBM5 (A and B) and CjCBM73 (C and D) incubated with $\alpha$-chitin for 60 min. The experiments were carried out at $22{ }^{\circ} \mathrm{C}$ using $10 \mathrm{~g} / \mathrm{L} \alpha$-chitin in $50 \mathrm{mM}$ sodium phosphate buffer $\mathrm{pH} 7.0$ and show binding of $C j \mathrm{CBM} 5$ and $C j \mathrm{CBM} 73$ to non-oxidized (A and $\mathrm{C}$ ) and oxidized (B and D) substrate. $\mathrm{P}_{\text {bound }}$ corresponds to bound protein ( $\mu \mathrm{moles} / \mathrm{g}$ substrate) and $\mathrm{P}_{\text {free }}$ corresponds to nonbound protein $(\mu \mathrm{M})$. The error bars show \pm s.d. $(n=3)$.

\section{Simulations provide insight into binding of CBMs to $\alpha$-chitin}

Coarse-grained (CG) simulations were performed to further investigate interactions between CBMs and a model of the surface of $\alpha$-chitin. CG models based on the Martini force-field represent 3-4 atoms by a single "bead", thereby reducing the number of particles that are simulated (29). This allows simulations to be run longer and to sample longer time scales, compared to atomistic simulations. We combined CG models of chitin and the CBMs with well-tempered metadynamics (WT-MetaD) simulations to further enhance sampling of CBM-chitin binding/unbinding events, which occur on long time scales. In the WT-MetaD approach, protein conformations along a set of collective variables are biased by a history-dependent potential. The total bias (i.e. sum of the Gaussians in the potential) forces the system to escape from local free-energy minima and explore different regions of the collective variable space. In the case of the CBM-chitin model we used two collective variables as proxies for binding: (i) the Euclidean distance $\left(r_{\text {chitin }}\right)$ and (ii) number of contacts, $\left\langle\mathrm{c}_{\mathrm{w}}\right\rangle$, between aromatic residues in the putative substrate binding surfaces (CjCBM5: Y282, W283, Y296; CjCBM73: W371, Y378, W386) and the chitin surface. Details on the calculation of these collective variables are provided in the Experimental procedures.

To promote binding to chitin by the CBMs it was necessary to rescale the interaction strengths between chitin beads and protein beads in the Martini model (see Experimental procedures for details). The effect of rescaling these interactions by $0 \%$ (unchanged) or by an up to $15 \%$ increase in the strength of the chitin-protein interaction was evaluated by running umbrella-sampling 
simulations (Figure S3) on the rescaled models, and by comparing dissociation constants calculated from these simulations with experimentally determined values (Figure 6). The results (Table 1) show that the best agreement with experiment was attained with a $10 \%$ increase in the chitin-protein interaction strength. The free-energy surfaces of $C j C B M 5$ and $C j C B M 73$ have similar appearances, but $C j \mathrm{CBM} 73$ has a deeper well than $C j \mathrm{CBM} 5$, which correlates with its experimentally observed stronger affinity for chitin (Figure S3).

The number of contacts between all amino acids in each CBM and the $\alpha$-chitin surface was calculated for every frame $(n=10000)$ in the WT-MetaD simulation and reweighted using the bias from the simulation (see Experimental procedures for details). The results (Figure 7) show which residues have the most contacts, i.e. $\left\langle\mathrm{c}_{\mathrm{w}}\right\rangle>0.5$, with the substrate over time. In the case of CjCBM5 (Figure 7A,C), regions with most contacts include, and are to a large extend limited to, the three aromatic residues of the putative binding surface (Y282, W283, Y296). Additionally, the region around Y265 seems to be somewhat involved in substrate binding albeit with much fewer contacts. These observations are in good agreement with the chemical shift perturbation data for binding of (GlcNAc)6. Similar observations were made for $C j C B M 73$ (Figure 7B,D), in the sense that also in this case the interacting regions include, and are to a large extend limited to, the three aromatic residues of the putative binding surface (W371, Y378, W386). Furthermore, also in this case interactions with fewer contacts $\left(0.2<\left\langle\mathrm{c}_{\mathrm{w}}\right\rangle<0.5\right)$ with a fourth aromatic residue, Y351, were observed. In order to match the experiments as closely as possible, we included the Cterminal His in the simulations and found that these have a number of contacts with the substrate. (Figure 7A,B) . All in all, these analyses show that the amino acids on the surface of CjCBM5 that most frequently interact with chitin form a relatively linear arrangement (Figure 7E), perhaps reflecting that interactions are limited to a single chitin chain, whereas the arrangement of aromatic amino acids on the surface of $\mathrm{CjCBM} 73$ is wider and suggests a more extended substrate-binding surface (Figure 7F). 

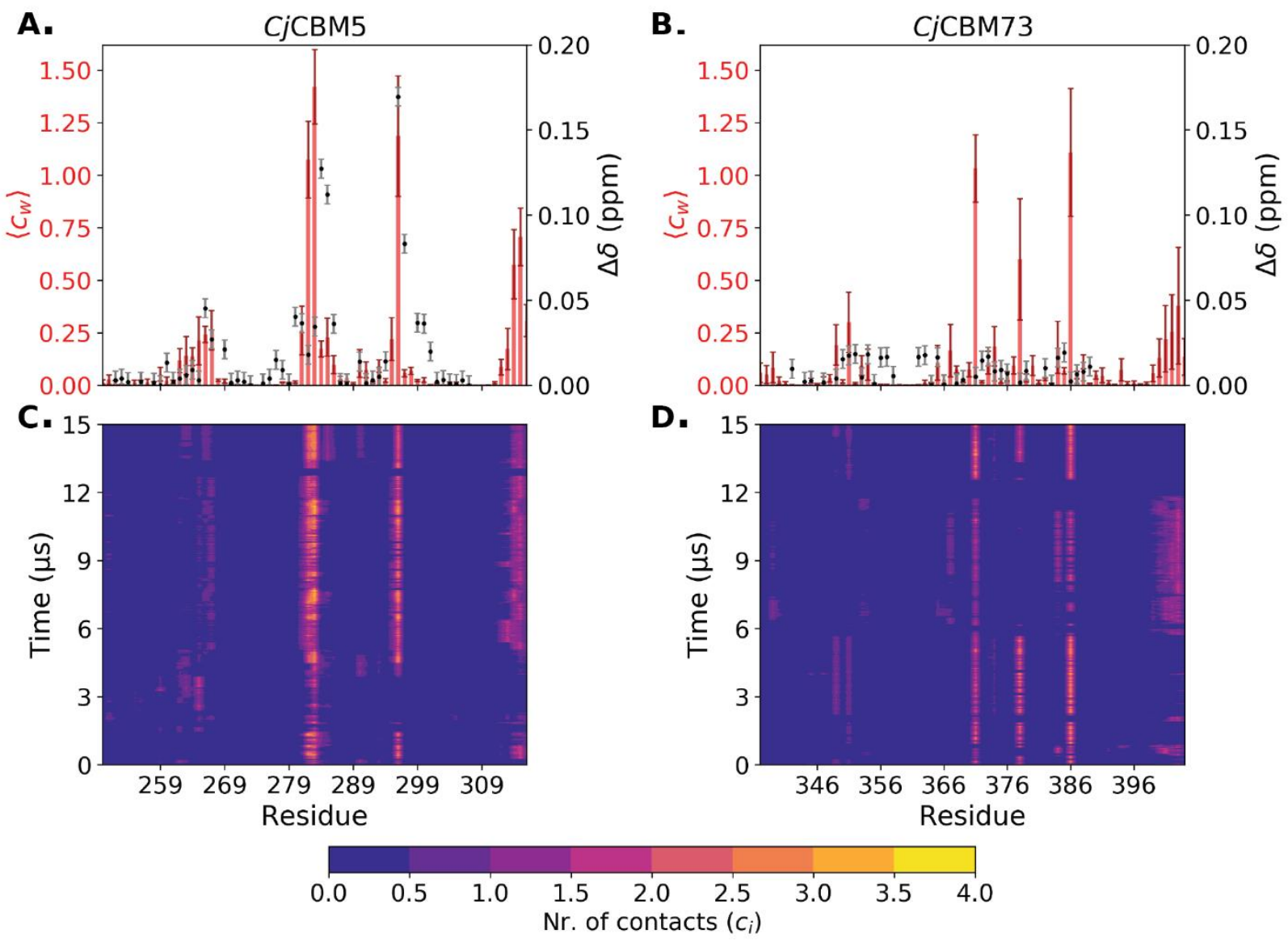

E.

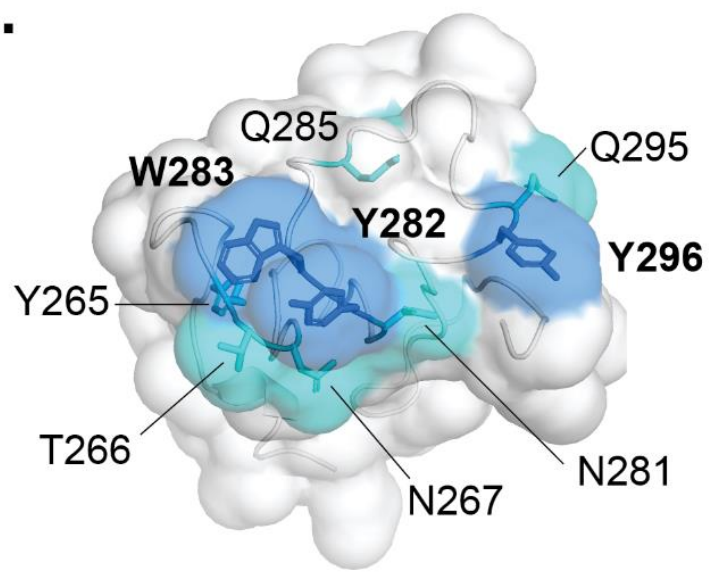

F.

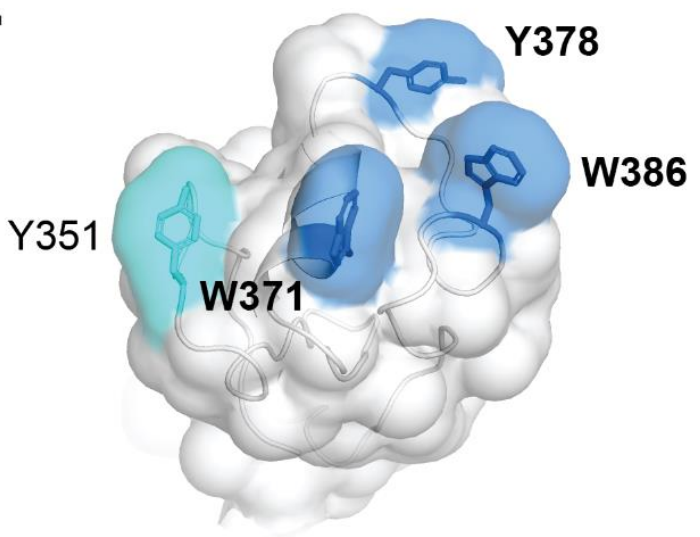

Figure 7. Chitin binding probed by NMR and simulations for CjCBM5 and CjCBM73. Panels A and $\mathrm{B}$ show the weighted average number of contacts observed during the simulation $\left(\langle c\rangle_{w}\right.$; red bars with dark red error bars) and the chemical shift perturbations ( $\Delta \delta$; black dots with grey error bars) observed by NMR upon addition of (GlcNAc)6. The error bars for the number of contacts were calculated using block analysis (30); error bars for chemical shift perturbations correspond to $0.003 \mathrm{ppm}$. Note that no significant chemical shift perturbations were recorded for $C j \mathrm{CBM} 73$. Panels $\mathrm{C}$ and $\mathrm{D}$ show the number of contacts between each amino acid and the $\alpha$-chitin surface per frame of the $15 \mu \mathrm{s}$ simulations, $c_{i}$, using a cut-off distance of $0.3 \mathrm{~nm}$ (see Experimental procedures for details). We note that due to the use of coarse-grained models and because of the use of 
metadynamics, i.e. enhanced sampling, the timescales do not here correspond to a physical time scale. Panels $\mathrm{E}$ and $\mathrm{F}$ show the substrate-binding surfaces of representative conformations of the bound state of $C j \mathrm{CBM} 5$ and $C j \mathrm{CBM} 73$, respectively. The side chains of amino acids on the binding surface that have most contacts $\left(\left\langle c_{w}\right\rangle>0.5\right)$ with chitin are colored blue, while the side chains of amino acids with fewer contacts $\left(0.2<\left\langle c_{w}\right\rangle<0.5\right)$ are colored cyan.

Table 1. Dissociation constants $\left(K_{\mathrm{d}}\right)$ for binding of the CjCBMs to $\alpha$-chitin, determined by experiments (see Figure 6) and simulations (see Figures 7 and S3). The modeled values were calculated from umbrella sampling simulations in which the interaction strength between chitin beads and protein beads remained unchanged $(0 \%)$ or was increased by $5 \%, 10 \%$ and $15 \%$.

\begin{tabular}{cccccc}
\hline \multirow{2}{*}{ Protein } & \multicolumn{4}{c}{ Simulations $(\boldsymbol{\mu M})$} & \multirow{2}{*}{ Experiments $(\boldsymbol{\mu M})$} \\
\cline { 2 - 5 } & $0 \%$ & $5 \%$ & $10 \%$ & $15 \%$ & \\
\hline CjCBM5 & 1800 & 480 & 36 & 0.65 & $8.9 \pm 0.5$ \\
$C j$ CBM73 & 290 & 76 & 15 & 0.13 & $2.9 \pm 0.1$ \\
\hline
\end{tabular}

\section{Discussion}

In multi-modular LPMOs, CBMs tethered to the LPMO domain have significant impact on the catalytic efficiency of the enzyme $(19,20,22,31)$. Therefore, it is important to gain a deeper understanding of the mechanisms by which CBMs recognize and bind their target substrates. Here, we have investigated two CBMs from $C j \mathrm{LPMO} 10 \mathrm{~A}, C j \mathrm{CBM} 5$ and $C j \mathrm{CBM} 73$, to illuminate structural and functional differences between these chitin-binding domains. The present results include the first structure for a member of the CBM73 family.

The NMR solution structures show that, although both CBMs have similar overall folds, $C j \mathrm{CBM} 73$ has a 310-helix connected by an additional disulfide bridge. These features appear to be conserved in CBM73 family (Figure S2). To obtain further insight into the structural variation between small chitin-binding CBMs, we compared the structures of $C j \mathrm{CBM} 5$ and $C j \mathrm{CBM} 73$ with the structures of five CBM5s and a CBM12 (Figure 8). The CBM12 is included since the CBMs in this family are closely related to family 5 CBMs (16). It has previously been shown (32-34) that, in addition to conserved surface-exposed aromatic residues, these CBMs share two additionally conserved aromatic amino acids (Y265 and W299 in CjCBM) that also occur in CBM73s (Y351 and W390 in $C j C B M 73$; Figure S2). These residues are a part of the hydrophobic core of the proteins. All CBMs (32-38) in Figure 8 bind chitin.

Previous studies $(33,34)$ have established the importance of the two consecutive, conserved aromatic residues, Y-W or W-W, in family 5 CBMs (Y282-W283 in CjCBM5). Site-directedmutagenesis studies have shown that a third aromatic residue, Y296, present on the surface of CjCBM5, PfChiA_CBM5 and MmChi60_CBM5, also contributes to chitin-binding (34). Whereas the NMR titration experiment with soluble chitohexaose did not show binding for CjCBM73, results for CjCBM5 showed that both W283 and Y296 are involved in binding (GlcNAc)6. Additionally, the polar residues T284 and Q285 also appear to contribute to binding (GlcNAc)6. These observations suggest that chitin-binding by CjCBM5 likely involves a combination of $\mathrm{CH}-\pi$ interactions (28) and hydrogen bonding. This is in good agreement with previous observations by Akagi et al. (33) who studied binding of to (GlcNAc) 6 to $\mathrm{SgChiC} C \mathrm{CBM}$. 
Like $C j$ CBM5 $\left(K_{\mathrm{d}}=2 \pm 1 \mathrm{mM}\right), S g C h i C \_C B M 5$ binds $(\text { GlcNAc })_{6}$ with low mM affinity $\left(K_{\mathrm{d}}=1.6\right.$ $\pm 0.3 \mathrm{mM})$.

CjCBM5, like other CBM5s in Figure 8, has three exposed aromatic residues with a close to linear arrangement of the side chains on the surface. This type of arrangement is often found in cellulosebinding domains (39-41), where the distance between the three aromatic residues coincides with the spacing of every second glucose ring in a single chain $(40,42)$. Compared to the other CBMs in Figure 8, the arrangement of the three exposed aromatic residues in $C j \mathrm{CBM} 73$; W371, Y378 and W386, differs, which suggests that $C j \mathrm{CBM} 73$ has a wider binding surface that may interact with several chitin chains. This could explain why $C j \mathrm{CBM} 73$ cannot bind (GlcNAc)6, a singlechain analog, while $C j \mathrm{CBM} 5$ can.
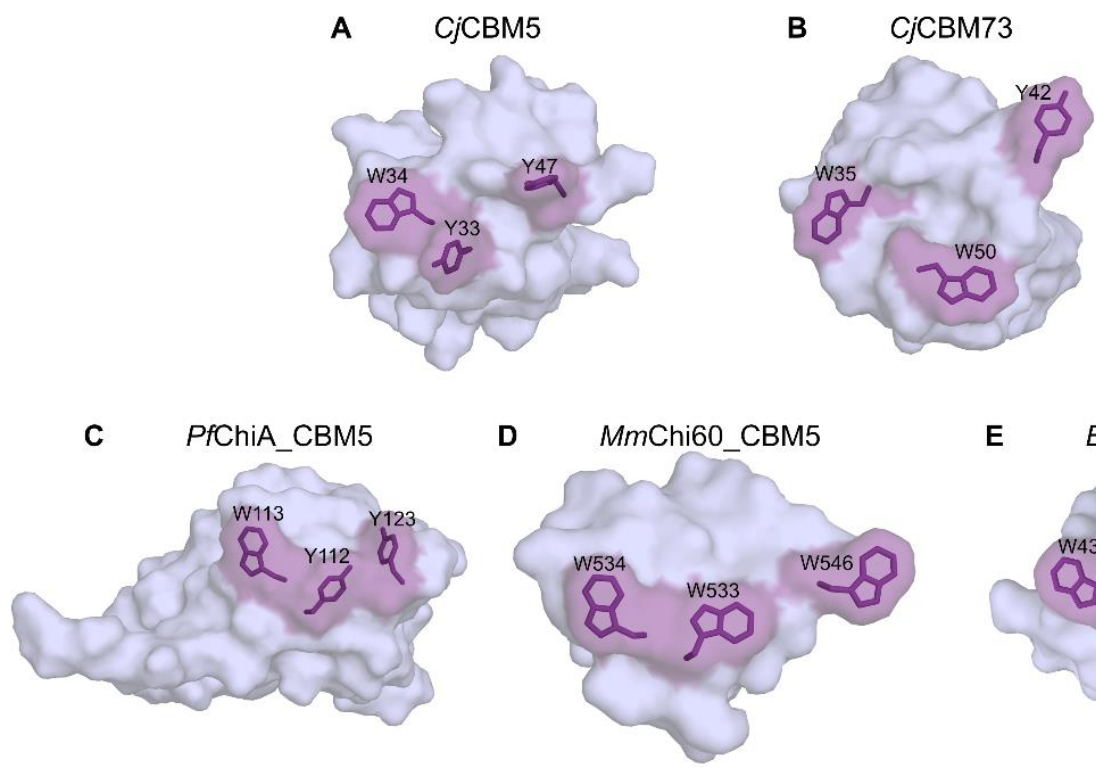

E EcEGZ_CBM5

$\mathbf{F}$

G
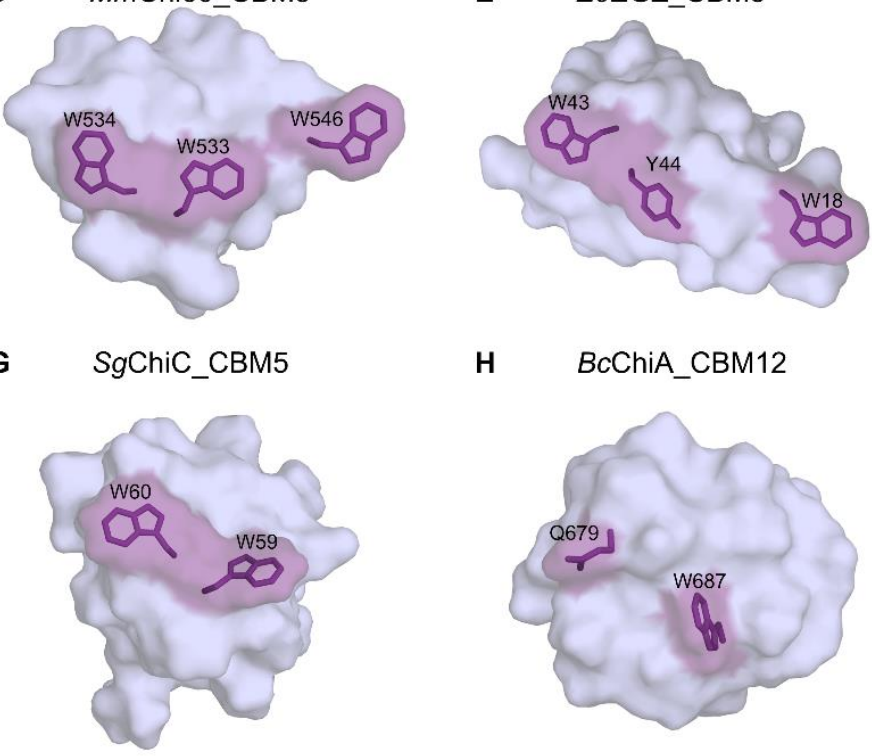

H

BcChiA_CBM12
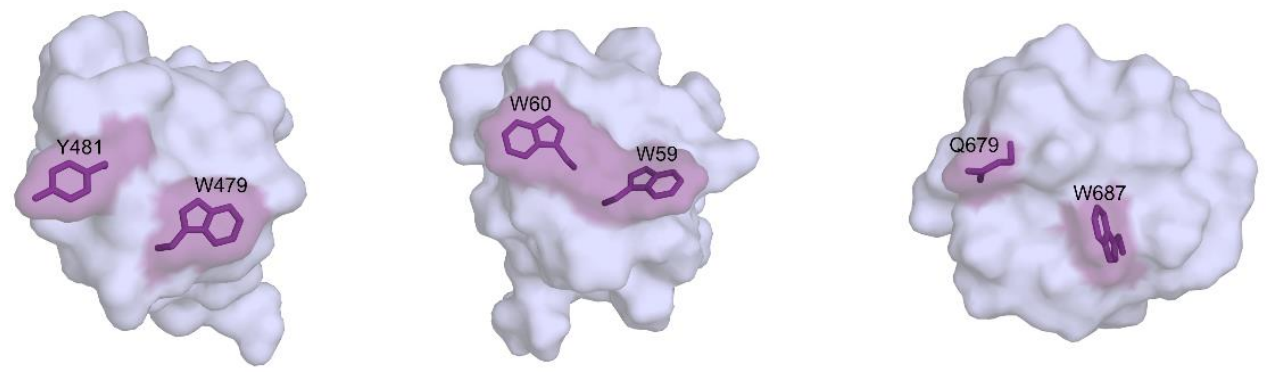

Figure 8. Comparison of the binding surfaces of the NMR structures of CjCBM5 and CjCBM73 with the structures of other CBM5 domains and one CBM12 domain. The other structures are derived from: PfChiA_CBM5 (34) (PDB ID: 2RTS; NMR structure), MmChi60_CBM5 (37) (PDB ID: 4HMC; X-ray diffraction structure), EcEGZ_CBM5 (27) (PDB ID: 1AIW; NMR structure), SmChiB_CBM5 (36) (PDB ID: 1E15; X-ray diffraction structure), SgChiC_CBM5 (33) (PDB ID: 2D49; NMR structure) and BcChiA_CBM12 $(32,38)$ (PDB ID: 1ED7; NMR structure). Residues shown or predicted to be involved in substrate binding are highlighted in purple. 
The distinct arrangements of amino acids on the binding surfaces of $C j \mathrm{CBM} 5$ and $C j \mathrm{CBM} 73$ may also explain the experimentally and computationally observed differences in binding to $\alpha$-chitin (Figures 6 and 7). The side chains of the amino acids with most chitin contacts in the simulations (Figure 7E) form a linear arrangement in CjCBM5 but are distributed on a larger and wider surface in $C j C B M 73$ (Figure 7F). Both experiment and simulations indicated that CjCBM5 binds to chitin with lower affinity compared to CjCBM73 (Table 1), whereas this is the other way around for (single chain) (GlcNAc)6. The stronger affinity of CjCBM73 for insoluble $\alpha$-chitin can be explained by its binding surface covering a larger area than the binding surface of $C j \mathrm{CBM} 5$.

At low substrate concentrations, the catalytic performance of $C j L P M O 10 A^{\mathrm{FL}}$ and $C j \mathrm{LPMO} \mathrm{A}^{\triangle \mathrm{CBM} 73}$ is superior to that of $C j \mathrm{LPMO} 10 \mathrm{~A}^{\mathrm{CD}}$ and the progress curves in Figure $2 \mathrm{~A}-\mathrm{B}$ show that this is due to rapid inactivation of $C j \mathrm{LPMO} \mathrm{AA}^{\mathrm{CD}}$. Forsberg et al (20) have previously shown that almost all the binding affinity for chitin in $C j L P M O 10 \mathrm{~A}^{\mathrm{FL}}$ resides on the CBMs. The strong binding provided by the CBMs ensures that the LPMO stays close to its substrate, thus increasing the chances that the interaction of the reduced catalytic domain with the oxygen cosubstrate leads to a productive reaction (i.e., cleavage of chitin) rather than futile turnover that may lead to auto-catalytic enzyme inactivation (43), as has previously been observed for other CBMcontaining LPMOs $(22,31)$. At the highest substrate concentration (Figure 2C), the efficiency of all enzyme variants was approximately the same, likely because the high substrate load favors $C j L P M O 10 A^{\mathrm{CD}}$ binding to chitin, reducing the frequency of futile turnovers and the concurrent risk of enzyme inactivation. This observation is in agreement with a previous study (22) showing that the negative effect of truncation of the CBM2 from a two-domain cellulose-active LPMO was smaller at higher substrate concentrations.

The protective effect of the substrate, mediated by the CBMs, became more evident at higher temperatures (Figure 3), where $C j \mathrm{LPMO} 10 \mathrm{~A}^{\mathrm{FL}}$ showed higher catalytic performance than $C j \mathrm{LPMO} \mathrm{A}^{\Delta \mathrm{CBM} 73}$, indicating that $C j \mathrm{CBM} 73$ appears to provide additional protection to the enzyme from thermal inactivation. It is conceivable that the increased performance at higher temperatures translates into increased performance at lower, more physiologically relevant temperatures where the enzyme may experience other types of stress, such as very low substrate concentrations or high levels of oxidant.

The abovementioned previous study with a two-domain cellulose LPMO (22) shows that the anchoring effect of the CBMs leads to a higher fraction of soluble oxidized products relative to oxidized sites on the insoluble substrate. A similar effect was also observed for $C j \mathrm{LPMO} 10 \mathrm{~A}^{\mathrm{FL}}$ and $C j \mathrm{LPMO} 10 \mathrm{~A}^{\triangle \mathrm{CBM} 73}$ which produced a higher fraction of soluble oxidized products compared

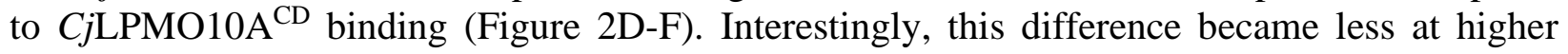
substrate concentrations, which is likely due to the fact that higher substrate concentrations increase the chance that a substrate-anchored but otherwise freely moving (22) catalytic domain acts on a neighboring fibril rather than the fibril to which it is bound.

Considering the LPMO reaction cycle and considering that anchoring by the CBMs could lead to multiple oxidized sites localized on the chitin surface around the CBM binding site, it is conceivable that accumulation of oxidized sites could trigger unbinding of the CBMs. The results of our attempts to test this hypothesis by studying CBM binding to partially oxidized chitin (Figure 6) were not conclusive, but did indicate that substrate oxidation slightly weakened chitin binding by $\mathrm{CjCBM} 5$. 
In conclusion, we have revealed structural and functional variation between the two chitin-binding domains in $C j$ LPMO10A. While it is clear that the presence of these CBMs has a significant effect on the catalytic performance of the LPMO, the reason for why nature has evolved enzymes with two slightly different chitin-binding domains remains unclear. It is conceivable that the combination of these domains provides advantages when acting on natural chitin-rich substrates, which likely are complex co-polymeric structures that may show structural variations. It is possible that functional differences between the CBM5 and the CBM73 remain undetected in the present experiments with a rather homogeneous, heavily processed chitin. Eventually, insights into these two CBMs will increase our understanding of how LPMOs depolymerize insoluble polysaccharides.

\section{Experimental procedures}

\section{Cloning, expression and purification of CjLPMO10A variants}

The gene encoding $C j \mathrm{LPMO}_{10 \mathrm{~A}^{\mathrm{FL}}}$ (residue 1-397) was codon optimized for E. coli expression. $C j$ LPMO $10 A^{\mathrm{CD}}$ (residue 1-216) was cloned into the pRSET B expression vector (Invitrogen) as previously described (20), as well as the construct lacking the CBM73 and the preceding polyserine linker, named $C j \mathrm{LPMO} \mathrm{A}^{\Delta \mathrm{CBM} 73}$ (residue 1-307).

To obtain better expression of $C j \mathrm{LPMO} 10 \mathrm{~A}^{\mathrm{FL}}$, the codon optimized gene encoding mature $C j$ LPMO $10 A^{\mathrm{FL}}$ (residues 37-397) was cloned behind an IPTG-inducible T5 promoter in the pD441-CH expression vector by ATUM (Newark, CA, USA), resulting in a fusion construct with an N-terminal E. coli OmpA signal peptide and a C-terminal His6 motif (Gly-(His)6). The expression vector was transformed into chemically competent E. coli BL21 (New England

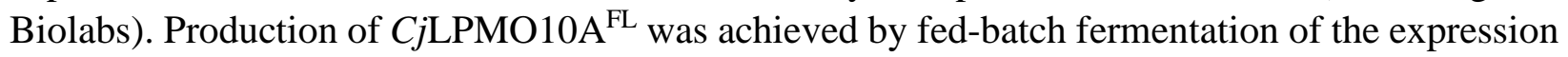
strain in a 1-liter fermenter (DASGIP® benchtop bioreactors for cell culture; Eppendorf, Hamburg, Germany), essentially as described previously (44), with the following modifications: at the start of the feed phase, the temperature was switched to $25{ }^{\circ} \mathrm{C}$, and $0.6 \mathrm{mM}$ isopropyl- $\beta$-Dthiogalactopyranoside (IPTG) was added to the glucose feed solution for continuous induction of gene expression. After $18 \mathrm{~h}$ of glucose feed, the cells were removed by centrifugation. The culture supernatant containing the target protein was concentrated 3-fold and buffer exchanged against 6 volumes of working buffer $(50 \mathrm{mM}$ Tris/ $\mathrm{HCl}, 300 \mathrm{mM} \mathrm{NaCl}, \mathrm{pH} 8.0)$ by crossflow filtration (Millipore Pellicon 2 mini filter, regenerated cellulose, $3 \mathrm{kDa} \mathrm{MWCO}$ ). After centrifugation for $30 \mathrm{~min}$ at $35,000 \mathrm{x} \mathrm{g}$ to remove precipitated proteins and filtration through a $0.2 \mu \mathrm{m}$ Nalgene Rapid-Flow sterile bottle-top filter unit (Thermo Scientific, Waltham, MA, USA), the culture filtrate was applied to a 20-mL nickel-nitrilotriacetic acid-sepharose column connected to an ÄKTA express FPLC system (GE Healthcare Lifesciences). After washing with 10 column volumes of working buffer containing $20 \mathrm{mM}$ imidazole, bound protein was eluted with a buffer containing $200 \mathrm{mM}$ imidazole. Fractions containing the target protein were pooled and buffer exchanged into $20 \mathrm{mM}$ Tris/HCl, $200 \mathrm{mM} \mathrm{NaCl}$, pH 7.5 by gel filtration over Sephadex G25 (GE Healthcare, 4 x HiPrep Desalting 26/10 columns).

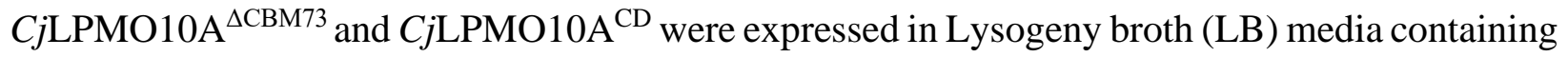
$50 \mu \mathrm{g} / \mathrm{mL}$ ampicillin. Cells harboring the plasmid was grown at $30{ }^{\circ} \mathrm{C}$ for $24 \mathrm{~h}$, without any 
induction, prior to harvest. The protein was extracted from the periplasmic space using an osmotic shock method that was first described by Manoil \& Beckwith (45), followed by purification using a two-step chromatography protocol. The periplasmic extract was adjusted to $50 \mathrm{mM}$ Tris/ $\mathrm{HCl} \mathrm{pH}$ 9.0 (loading buffer) and loaded onto a $5 \mathrm{~mL}$ Q Sepharose anion exchange column (GE Healthcare). Proteins were eluted using a linear salt gradient $(0-500 \mathrm{mM} \mathrm{NaCl})$ over 60 column volumes using a flow rate of $2.5 \mathrm{~mL} / \mathrm{min}$. LPMO containing fractions were pooled and concentrated to $1 \mathrm{~mL}$ before being loaded onto a HiLoad 16/60 Superdex 75 size exclusion column (GE Healthcare) operated with a running buffer consisting of $50 \mathrm{mM}$ Tris $\mathrm{pH} 7.5$ and $200 \mathrm{mM} \mathrm{NaCl}$, at a flow rate of $1 \mathrm{~mL} / \mathrm{min}$. Fractions containing pure LPMO were identified by SDS-PAGE and subsequently pooled and concentrated using Amicon Ultra centrifugal filters (Millipore) with a molecular weight cut-off of $10 \mathrm{kDa}$. Protein concentrations were measured using the Bradford assay (Bio$\mathrm{Rad})$. The protein solutions were stored at $4{ }^{\circ} \mathrm{C}$ until further use.

Expression plasmids for CjCBM5 (residue 251-309) and CjCBM73 (residue 338-397) based on the pNIC-CH vector (Addgene) were used for cytoplasmic expression as previously described (20). This cloning procedure adds a Met residue to the N-terminus as well as one Ala residue and a poly-histidine tag (6xHis-tag) to the C-terminus of both proteins. Pre-cultures in $5 \mathrm{~mL} \mathrm{LB}$ medium $(10 \mathrm{~g} / \mathrm{L}$ tryptone, $5 \mathrm{~g} / \mathrm{L}$ yeast extract and $5 \mathrm{~g} / \mathrm{L} \mathrm{NaCl})$ were used to inoculate $500 \mathrm{~mL}$ of TB-medium (Terrific Broth) supplemented with $50 \mu \mathrm{g} / \mathrm{mL}$ kanamycin. The cultures were grown at $37{ }^{\circ} \mathrm{C}$ for approximately 3 hours in a LEX-24 Bioreactor (Harbinger Biotechnology, Canada) using compressed air for aeration and mixing. Expression was induced by adding IPTG to a final concentration of $0.1 \mathrm{mM}$ at an optical density at $600 \mathrm{~nm}\left(\mathrm{OD}_{600}\right)$ of 0.6 , followed by incubation for $24 \mathrm{~h}$ at $23^{\circ} \mathrm{C}$. Cells were harvested by centrifugation $(5,500 \times \mathrm{g}, 10 \mathrm{~min})$ followed by cell lysis using pulsed sonication in a buffer containing $50 \mathrm{mM}$ Tris/ $\mathrm{HCl} \mathrm{pH} 8.0,500 \mathrm{mM} \mathrm{NaCl}$ and $5 \mathrm{mM}$ imidazole. Cell debris was removed by centrifugation $(75,000 \times \mathrm{g}, 30 \mathrm{~min})$ and the supernatant was loaded onto a $5 \mathrm{~mL}$ HisTrap HP Ni Sepharose column (GE Healthcare) equilibrated with lysis buffer. The protein was eluted by applying a $25 \mathrm{CV}$ linear gradient to reach $100 \%$ of a buffer containing $50 \mathrm{mM}$ Tris/ $\mathrm{HCl} \mathrm{pH} 8.0,500 \mathrm{mM} \mathrm{NaCl}$ and $500 \mathrm{mM}$ imidazole, at a flow rate of 2.5 $\mathrm{mL} / \mathrm{min}$. Protein containing fractions were analyzed by SDS-PAGE and subsequently concentrated, with concomitant buffer exchange to $20 \mathrm{mM}$ Tris/ $\mathrm{HCl} \mathrm{pH} \mathrm{8.0,} \mathrm{using} \mathrm{an} \mathrm{Amicon®}$ Ultra centrifugal filter (Millipore) with $3 \mathrm{kDa}$ cut-off. The concentrations of CjCBM5 and CjCBM73 were determined by measuring $\mathrm{A}_{280}$ and calculated using theoretical molar extinction coefficients $\left(\varepsilon_{280, C j \mathrm{CBM} 5}=22,585 \mathrm{M}^{-1} \mathrm{~cm}^{-1}, \varepsilon_{280, C j \mathrm{CBM} 73}=28,210 \mathrm{M}^{-1} \mathrm{~cm}^{-1}\right)$.

\section{Production of CjCBM5 and CjCBM73 for NMR studies}

CjCBM5 and CjCBM73 samples for NMR studies were produced both with ${ }^{13} \mathrm{C}$ and ${ }^{15} \mathrm{~N}$ isotopic labeling and ${ }^{15} \mathrm{~N}$ labeling only. A preculture was grown in $6 \mathrm{~mL} \mathrm{LB}$ medium supplemented with $50 \mu \mathrm{g} / \mathrm{mL}$ kanamycin in a shaking incubator at $30{ }^{\circ} \mathrm{C}, 225 \mathrm{rpm}$, for six hours. A main culture of $500 \mathrm{~mL} \mathrm{M} 9$ medium $\left(6 \mathrm{~g} / \mathrm{L} \mathrm{Na}_{2} \mathrm{HPO}_{4}, 3 \mathrm{~g} / \mathrm{L} \mathrm{KH}_{2} \mathrm{PO}_{4}, 0.5 \mathrm{~g} / \mathrm{L} \mathrm{NaCl}\right)$ supplemented with 500 $\mu \mathrm{g} / \mathrm{mL}$ kanamycin, $0.5 \mathrm{~g}\left({ }^{15} \mathrm{NH}_{4}\right)_{2} \mathrm{SO}_{4}, 6 \mathrm{~mL}$ glycerol, $5 \mathrm{~mL}{ }^{15} \mathrm{~N}$ Bioexpress Cell Growth Medium (Cambridge Isotope Laboratories, Tewksbury, MA, USA), $5 \mathrm{~mL}$ of Gibco MEM Vitamin Solution $(100 \mathrm{x}), 1 \mathrm{~mL} \mathrm{MgSO}_{4}(1 \mathrm{M})$ and $5 \mathrm{~mL}$ of a trace-metal solution $\left(0.1 \mathrm{~g} / \mathrm{L} \mathrm{ZnSO}_{4}, 0.8 \mathrm{~g} / \mathrm{L} \mathrm{MnSO}_{4}\right.$, $0.5 \mathrm{~g} / \mathrm{L} \mathrm{FeSO}_{4}, 0.1 \mathrm{~g} / \mathrm{L} \mathrm{CuSO}_{4}, 1 \mathrm{~g} / \mathrm{L} \mathrm{CaCl}_{2}$ ) was inoculated with $1 \%$ of the preculture and incubated at $22{ }^{\circ} \mathrm{C}$ in a LEX-24 Bioreactor as described above. After 18 hours, the cultures were induced with $0.5 \mathrm{mM}$ IPTG to a final concentration of $0.5 \mathrm{mM}$ was followed by incubation at 22 
${ }^{\circ} \mathrm{C}$ for $24 \mathrm{~h}$. Cells were harvested by centrifugation at $4{ }^{\circ} \mathrm{C}, 6000 \times \mathrm{g}$, for $5 \mathrm{~min}$. The pellet was resuspended in $20 \mathrm{~mL}$ lysis buffer $(50 \mathrm{mM}$ Tris- $\mathrm{HCl}, 50 \mathrm{mM} \mathrm{NaCl}, 0.05 \%$ Triton $\mathrm{X}-100, \mathrm{pH} 8.0$ ) supplemented with 1 tablet EDTA-free cOmplete ULTRA protease inhibitor (Roche) followed by pulsed sonication. Cell debris was removed by centrifugation at $4{ }^{\circ} \mathrm{C}, 16,600 \times \mathrm{g}$, for $45 \mathrm{~min}$. The supernatant was sterilized by filtration through a $0.2 \mu \mathrm{m}$ Sterile-flip filter unit (Nalgene). Buffer $\mathrm{B}(50 \mathrm{mM}$ Tris- $\mathrm{HCl}, 400 \mathrm{mM}$ imidazole, $\mathrm{pH}$ 8.0) was added to the filtered lysate to obtain a final concentration of $20 \mathrm{mM}$ imidazole. The proteins were purified by loading the supernatant onto a $1 \mathrm{~mL}$ HisTrap HP Ni-sepharose column (GE Healthcare Life Sciences) equilibrated with $5 \mathrm{CV}$ of 95\% Buffer A (50 mM Tris- $\mathrm{HCl}, \mathrm{pH} \mathrm{8.0)}$ ) and $5 \%$ Buffer B with a flow rate of $1 \mathrm{~mL} / \mathrm{min}$. Impurities were removed by washing with $95 \%$ Buffer A and $5 \%$ Buffer B for $10 \mathrm{CV}$. The protein was eluted using a $30 \mathrm{CV}$ gradient of 5-100\% Buffer B. The purity of the protein fractions was assessed with SDS-PAGE.

The protocol for production and purification of non-labeled samples of $C j \mathrm{CBM} 5$ and $C j \mathrm{CBM} 73$ for NMR studies was done as described above, except that 2xLB medium (20 g/L tryptone, $10 \mathrm{~g} / \mathrm{L}$ yeast extract and $5 \mathrm{~g} / \mathrm{L} \mathrm{NaCl}$ ) was used instead of $\mathrm{M} 9$.

Fractions shown to contain $C j \mathrm{CBM} 73$ were pooled and concentrated using Amicon Ultra protein concentrators $\left(\mathrm{MWCO} 3 \mathrm{kDa}\right.$ ) at $10{ }^{\circ} \mathrm{C}$ and $7000 \times \mathrm{g}$ to obtain a volume of $\sim 5 \mathrm{~mL}$. This protein solution was loaded onto a size-exclusion chromatography (SEC) column (HiLoad 16/600 Superdex $75 \mathrm{pg} ; 120 \mathrm{~mL} \mathrm{CV}$ ) that had been equilibrated with SEC-buffer pH 7.5 (50 mM Tris$\mathrm{HCl}, 20 \mathrm{mM} \mathrm{NaCl}$ ) for $1 \mathrm{CV}$. Protein fractions were eluted using a $1 \mathrm{~mL} / \mathrm{min}$ flow rate and the concentration was measured as mentioned above.

The buffer in the protein-containing fractions was exchanged to NMR buffers (for structure elucidation: $25 \mathrm{mM}$ sodium phosphate and $10 \mathrm{mM} \mathrm{NaCl}, \mathrm{pH}$ 5.5; for interaction studies: $50 \mathrm{mM}$ sodium phosphate (CjCBM5) or $25 \mathrm{mM}$ sodium phosphate (CjCBM73), pH 7.0) prior to concentrating to $\sim 70 \mu \mathrm{M}$ and a final volume of $\sim 400 \mu \mathrm{L}$. All steps were performed by centrifugation using Amicon Ultra protein concentration tubes (MWCO $3 \mathrm{kDa}$ ) at $10{ }^{\circ} \mathrm{C}$ and 7000 $\times \mathrm{g}$. NMR samples were prepared by adding $\mathrm{D}_{2} \mathrm{O}$ to a final ratio of $90 \% \mathrm{H}_{2} \mathrm{O} / 10 \% \mathrm{D}_{2} \mathrm{O}$.

\section{Chitin degradation experiments}

Unless stated otherwise, reactions were performed with $0.5 \mu \mathrm{M}$ LPMO in $50 \mathrm{mM}$ sodium phosphate buffer $\mathrm{pH} 7.0$ in the presence of $1 \mathrm{mM}$ ascorbic acid at $37{ }^{\circ} \mathrm{C}$ and $800 \mathrm{rpm}$ in an Eppendorf thermomixer. All reactions were performed in triplicates. 


\section{Preparation of oxidized chitin for binding studies}

$C j$ LPMO10A ${ }^{\mathrm{CD}}$ which is known to bind weakly to $\alpha$-chitin (20) and which was expected to oxidize the chitin surface more randomly compared to the full length enzyme (Figure 1; (22)) was used to prepare oxidized chitin. Six $1 \mathrm{~mL}$ reactions, each containing $20 \mathrm{~g} / \mathrm{L} \alpha$-chitin suspended in $50 \mathrm{mM}$ sodium phosphate $\mathrm{pH} 7.0$, were supplemented with $1 \mu \mathrm{M} C \mathrm{CJPMO} 10 \mathrm{~A}^{\mathrm{CD}}$ and $1 \mathrm{mM}$ ascorbic acid three times with $24 \mathrm{~h}$ intervals (i.e. to a final concentration of $3 \mu \mathrm{M}$ enzyme and $3 \mathrm{mM}$ ascorbic acid). The reactions were incubated in a thermomixer set to $37{ }^{\circ} \mathrm{C}$ and $800 \mathrm{rpm}$. After $72 \mathrm{~h}$ of incubation, samples were taken from all six reactions and diluted in buffer supplemented with 2 $\mu \mathrm{M} S m \mathrm{ChiA}(46)$ and $0.5 \mu \mathrm{M} \operatorname{SmCHB}(47)$ to a substrate concentration of $2 \mathrm{~g} / \mathrm{L}$. These reaction mixtures were incubated for $24 \mathrm{~h}$ at $37^{\circ} \mathrm{C}$ at $800 \mathrm{rpm}$ after which oxidized products were analyzed quantitatively to determine the total degree of oxidation in the LPMO-treated chitin. The rests of the $20 \mathrm{~g} / \mathrm{L}$ reactions were centrifuged in an Eppendorf centrifuge (13,000 rpm for $3 \mathrm{~min})$, the supernatant was removed and the soluble products in the supernatant were subjected to degradation with $2 \mu \mathrm{M}$ SmChiA and $0.5 \mu \mathrm{M} \operatorname{SmCHB}$ as described above, to determine the amount of solubilized oxidized products. The pelleted oxidized chitin was washed with buffer $(3 \times 1 \mathrm{~mL}$ of $50 \mathrm{mM}$ sodium phosphate $\mathrm{pH}$ 7.0) by repetitively suspending the chitin in buffer and removing the supernatant after centrifugation. Finally, the oxidized chitin was suspended in buffer to $20 \mathrm{~g} / \mathrm{L}$. Again, samples were taken from all six reactions and diluted in buffer supplemented with $2 \mu \mathrm{M}$ $S m \mathrm{ChiA}$ and $0.5 \mu \mathrm{M} S m \mathrm{CHB}$ to a substrate concentration of $2 \mathrm{~g} / \mathrm{L}$. The reactions were incubated for $24 \mathrm{~h}$ at $37{ }^{\circ} \mathrm{C}$ at $800 \mathrm{rpm}$ and the resulting samples were used to determine the amount of insoluble oxidized products). Compounds in $\mathrm{SmChiA} / \mathrm{SmCBH}$ degraded samples was quantified as described below.

\section{Quantitative analysis of GlcNAcGlcNAclA}

Prior to product quantification, LPMO-generated products, were degraded with only $S m \mathrm{CHB}$ (soluble fractions) or a combination with $S m$ ChiA and $S m \mathrm{CHB}$ (for total or insoluble fractions) to yield the oxidized dimer (GlcNAcGlcNAc1A) and non-oxidized monomer (GlcNAc). Analysis and quantification of chitobionic acid (GlcNAcGlcNAc1A) were carried out using an RSLC system (Dionex) equipped with a $100 \times 7.8 \mathrm{~mm}$ Rezex RFQ-Fast Acid H+ (8\%) (Phenomenex, Torrance, CA, USA) column operated at $85^{\circ} \mathrm{C}$. Samples of $8 \mu \mathrm{L}$ were injected to the column and sugars were eluted isocratically using $5 \mathrm{mM}$ sulphuric acid as mobile phase with a flow rate of 1 $\mathrm{mL} / \mathrm{min}$. Standards of GlcNAcGlcNAc1A $(10-500 \mu \mathrm{M})$ were used for quantification. GlcNAcGlcNAc1A was generated in-house by complete oxidation of $N$-acetyl-chitobiose (Megazyme; 95\% purity) by the Fusarium graminearum chitooligosaccharide oxidase ( $\mathrm{FgChitO}$ ) as previously described $(47,48)$.

\section{Determination of apparent melting temperature (Tm)}

The apparent melting temperature $\left(\mathrm{T}_{\mathrm{m}}\right)$ of the proteins was determined according to a protein thermal shift assay (ThermoFisher Scientific) based on using SYPRO orange, a fluorescent dye, to monitor protein unfolding (49). The quantum yield of the dye is significantly increased upon binding to hydrophobic regions of the protein that become accessible as the protein unfolds. The 
fluorescence emission (RFU) was monitored using a StepOnePlus real-time PCR machine (ThermoFisher Scientific). $\mathrm{T}_{\mathrm{m}}$ was calculated as the temperature corresponding to the minimum value of the derivative plot $(-\mathrm{d}(R F U) / \mathrm{dT}$ vs T; Figure $\mathrm{S} 2) .0 .1 \mathrm{~g} / \mathrm{L}$ LPMO in $50 \mathrm{mM}$ sodium phosphate buffer ( $\mathrm{pH} 7.0$ ) was heated in the presence of the dye in a 96-well plate from $25{ }^{\circ} \mathrm{C}$ to $95^{\circ} \mathrm{C}$, over 50 minutes. For each protein, the experiment was carried out in quadruplicates (i.e. $\mathrm{n}$ $=4)$.

\section{Binding studies with CjCBM5 and CjCBM73}

Binding studies were performed as previously described (20). The equilibrium binding constants $\left(K_{\mathrm{d}}\right)$ and binding capacity $\left(\mathrm{B}_{\max }\right)$ were determined for $C j \mathrm{CBM} 5$ and $C j \mathrm{CBM} 73$ by mixing protein solutions of varying concentrations $(0,20,50,75,100,150,300$ and $500 \mu \mathrm{g} / \mathrm{ml}$ for CjCBM5 and $0,10,20,50,75,100,150$, and $300 \mu \mathrm{g} / \mathrm{ml}$ for $C j C B M 73$; protein concentration determined by $\mathrm{A}_{280}$ ) with $10 \mathrm{mg} / \mathrm{ml}$ pre-oxidized (see above) or untreated $\alpha$-chitin. Before adding the chitin, $\mathrm{A}_{280}$ was measured for each of the prepared protein solutions (in $50 \mathrm{mM}$ sodium phosphate buffer, $\mathrm{pH}$ 7.0), to create individual standard curves for each protein. After addition of chitin, the solutions were placed at $22{ }^{\circ} \mathrm{C}$ in an Eppendorf Comfort Thermomixer set to $800 \mathrm{rpm}$ for $60 \mathrm{~min}$. Subsequently, samples were filtered using a 96-well filter plate (Millipore), and the concentration of free protein in the supernatant was determined by measuring $\mathrm{A}_{280}$. All assays were performed in triplicate and with blanks (buffer and $10 \mathrm{mg} / \mathrm{ml} \alpha$-chitin). The equilibrium dissociation constants, $K_{\mathrm{d}}(\mu \mathrm{M})$, and substrate binding capacities, $\mathrm{B}_{\max }(\mu \mathrm{mol} / \mathrm{g} \alpha$-chitin), were determined by fitting the binding isotherms to the one-site binding equation where $\mathrm{P}$ represents protein: $\left[P_{\text {bound }}\right]=B_{\max }\left[P_{\text {free }}\right] /\left(K_{d}+\left[P_{\text {free }}\right]\right)$, by nonlinear regression using the Prism 6 software (GraphPad, La Jolla, CA).

\section{NMR spectroscopy}

NMR spectra of $70 \mu \mathrm{M} C$ CjCBM5 and CjCBM73 in NMR buffer (25 mM sodium phosphate and $10 \mathrm{mM} \mathrm{NaCl}, \mathrm{pH} 5.5$ ) containing $10 \% \mathrm{D}_{2} \mathrm{O}$ were recorded at $25^{\circ} \mathrm{C}$ on a Bruker Ascend $800 \mathrm{MHz}$ spectrometer with an Avance III HD (Bruker Biospin) console equipped with a $5 \mathrm{~mm}$ Z-gradient CP-TCI (H/C/N) cryogenic probe at the NV-NMR-Centre/Norwegian NMR Platform at NTNU, the Norwegian University of Science and Technology, Trondheim, Norway. ${ }^{1} \mathrm{H}$ chemical shifts were referenced internally to the water signal, while ${ }^{13} \mathrm{C}$ and ${ }^{15} \mathrm{~N}$ chemical shifts were referenced indirectly to water based on the absolute frequency ratios (50). Backbone and side chain assignments of $C j \mathrm{CBM} 5$ and $C \mathrm{CJCBM} 73$ were obtained using ${ }^{15} \mathrm{~N}-\mathrm{HSQC},{ }^{13} \mathrm{C}-\mathrm{HSQC}$, HNCA, $\mathrm{HN}(\mathrm{CO}) \mathrm{CA}, \mathrm{HNCO}, \mathrm{HN}(\mathrm{CA}) \mathrm{CO}, \mathrm{CBCANHHNCACB}, \mathrm{CBCA}(\mathrm{CO}) \mathrm{NH}$ and $\mathrm{H}(\mathrm{C}) \mathrm{CH}-\mathrm{TOCSY}$. For CjCBM5 the BEST (band-selective excitation short-transient) (51) versions of HNCA, $\mathrm{HN}(\mathrm{CO}) \mathrm{CA}, \mathrm{HNCO}, \mathrm{HN}(\mathrm{CA}) \mathrm{CO}$ and $\mathrm{HN}(\mathrm{CO}) \mathrm{CACB}$ were recorded. The assignments have been deposited in the BioMagnetic Resonance Databank (BMRB) under the IDs 34519 (CjCBM5) and 34520 (CjCBM73). 


\section{Structure elucidation}

The NMR data were recorded and processed with TopSpin version 3.6 and analyzed with CARA version 1.5 .5 (52). For structure determination, 3D ${ }^{13} \mathrm{C}$-edited and ${ }^{15} \mathrm{~N}$-edited NOESY-HSQC spectra, as well as $2 \mathrm{D}{ }^{1} \mathrm{H}-{ }^{1} \mathrm{H}$ NOESY spectra were recorded. NOE cross-peaks were manually identified, assigned, and integrated using the NEASY program within CARA version 1.5.5. Dihedral torsion angles $(\phi$ and $\psi)$ were calculated from chemical shift data $\left(\mathrm{C}^{\alpha}, \mathrm{C}^{\beta}, \mathrm{H}^{\mathrm{N}}, \mathrm{H}^{\alpha}, \mathrm{H}^{\beta}, \mathrm{N}\right.$ and C') by TALOS-N (53). Structures were calculated using the torsion angle dynamics program CYANA 3.97 (54). The structure calculation started by generating 200 conformers with random torsion angles, and the dihedral angles in each conformer were optimized using simulated annealing in 10,000 steps, to fit the restraints. The 20 conformers with the lowest CYANA target function values were energy-minimized using YASARA (55), first in vacuo, followed by using water as the explicit solvent and calculating electrostatics by applying the particle mesh Ewald method (56). In both these steps the YASARA force field (57) was applied. The coordinates of the minimized CBM conformers have been deposited in the Protein Data Bank (PDB) under the IDs $6 Z 40$ (CjCBM5) and 6Z41 (CjCBM73). The two structures were aligned using the combinatorial extension (CE) algorithm, which determines the longest continuous alignment between fragment pairs (58).

\section{Titration of CBMs with chitohexaose}

The interaction between CjCBM5 and chitohexaose, $(\mathrm{GlcNAc})_{6}$, was investigated using NMR spectroscopy. A ${ }^{15} \mathrm{~N}$-HSQC spectrum was recorded of a sample of ${ }^{15} \mathrm{~N}$-labeled $C j \mathrm{CBM} 5(70 \mu \mathrm{M})$ in $50 \mathrm{mM}$ sodium phosphate containing $10 \% \mathrm{D}_{2} \mathrm{O}$ and used as reference. Another sample of ${ }^{15} \mathrm{~N}$ labeled $C_{j C B M}(70 \mu \mathrm{M})$ with $10 \mathrm{mM}$ (GlcNAc) 6 in $50 \mathrm{mM}$ sodium phosphate containing $10 \%$ $\mathrm{D}_{2} \mathrm{O}$ was prepared. After recording a ${ }^{15} \mathrm{~N}-\mathrm{HSQC}$ spectrum of this latter sample, it was mixed with the reference sample to obtain the following concentrations of (GlcNAc) 6 0.2, 0.5, 1.0 and 5.0 $\mathrm{mM}$, while maintaining a constant protein concentration. A new ${ }^{15} \mathrm{~N}-\mathrm{HSQC}$ spectrum was recorded at each (GlcNAc) . The chemical shift perturbations $(\Delta \delta)$ were calculated using the equation: $\Delta \delta=\sqrt{\left(\Delta \delta_{H}\right)^{2}+\left(\Delta \delta_{N} / 6.5\right)^{2}}$, where $\Delta \delta_{H}$ is the change in chemical shift for the amide proton and $\Delta \delta_{N}$ for the amide nitrogen, in ppm (59). The $K_{d}$ was estimated using Gnuplot 5.2 (www.gnuplot.info) based on an average of the amide chemical shift perturbation $(\Delta \delta)$ from the five most affected amino acids (W283, T284, Q285, Y296 and G297). The function used for fitting was $\Delta \delta=\Delta \delta_{\max }[S] /\left(K_{d}+[S]\right)$, where $\Delta \delta_{\max }$ describes the binding capacity as the maximum value of $\Delta \delta$. Error bars in the chemical shift measurements correspond to $0.003 \mathrm{ppm}$.

The same procedure was applied for the NMR titration of $C j \mathrm{CBM} 73$ with $(\mathrm{GlcNAc})_{6}$, using CjCBM73 $(500 \mu \mathrm{M})$ and the following (GlcNAc) ${ }_{6}$ concentrations: 0.2, 0.6, 1.2, 2.8, 5.6 and 6.5 $\mathrm{mM}$.

\section{Modelling coarse-grained $\alpha$-chitin}

The crystal structure of $\alpha$-chitin at $300 \mathrm{~K}(60)$ was used to generate an all-atom $\alpha$-chitin surface composed of 12 chains with 20 residues each, in UCSF Chimera version 1.13.1 (61). The all-atom model was coarse-grained using the bead mapping and topology parameters proposed by $\mathrm{Yu} \&$ Lau (62) and bead types from the coarse-grained Martini version 3.0.beta.4.17 force-field (29). A 
rectangular simulation box was defined with the same size as the surface in the $\mathrm{y}(110 \AA)$ and $\mathrm{z}$ (100 $\AA$ ) dimensions and $150 \AA$ in the $\mathrm{x}$ dimension.

\section{Coarse-grained simulations}

The coarse-grained Martini version 3.0.beta.4.17 force-field was used in combination with GROMACS 5.1.4 (63) to simulate interactions between the CBMs and $\alpha$-chitin. The constructs were coarse-grained using the Martinize2 program (64). An elastic network model (65) was used to constrain the overall structure of the CBMs. The beads in the chitin surface were kept in place by applying a harmonic potential with a force constant of $1000 \mathrm{~kJ} \mathrm{~mol}^{-1} \mathrm{~nm}^{-1}$ on the $\mathrm{x}$, $\mathrm{y}$ and $\mathrm{z}$ positions. Models of $C j \mathrm{CBM} 5$ or $C j \mathrm{CBM} 73$ were manually placed in the simulation box above the chitin surface by using PyMOL (66). The simulation box was filled with water beads and the system was neutralized with beads corresponding to $\mathrm{Na}^{+}$and $\mathrm{Cl}^{-}$ions to an ionic strength of 0.15 M. The complex was energy-minimized using a steepest-descent algorithm (100 steps, $0.03 \mathrm{~nm}$ max step size) prior to being relaxed for $1 \mathrm{~ns}$, with a time step of $5 \mathrm{fs}$, using the velocity-rescale (v-rescale) thermostat (67), Parrinello-Rahman barostat (68) and Verlet cutoff scheme (69). Simulations were run on the relaxed models with a time step of $20 \mathrm{fs}$, using the v-rescale thermostat, Parrinello-Rahman barostat and Verlet cutoff scheme in the NPT ensemble. Frames were written every $1 \mathrm{~ns}$ for each trajectory. Representative conformations of coarse-grained models of $C j \mathrm{CBM} 5$ and $C j \mathrm{CBM} 73$ were backmapped to atomistic models by using the CG2AT2 program (70).

\section{Adjusting the protein-chitin interaction strength in the Martini force-field}

Initially, binding between CBMs and chitin was not observed, therefore, drawing inspiration from Larsen et al (71), we considered the following approach to modify the Martini version 3.0.beta.4.17 force-field to promote CBM binding to chitin. First, the interaction strength (i.e. $\varepsilon$ parameter in the Lennard-Jones potential) between chitin beads and protein beads was increased by $10 \%$ and welltempered metadynamics simulations (WT-MetaD; see below) were run until binding between the CBMs and chitin was observed. Then, a binding path for each CBM, which included bound and unbound conformations was selected from the WT-MetaD simulations, and umbrella-sampling (US) simulations were performed on these conformations as described below. Finally, we tested different interaction strengths by generating topologies where the interaction strength was modified from $0 \%$ (unchanged) to $15 \%$ increase of the chitin-protein interaction strength and ran umbrella-sampling simulations for each interaction strength. Figure S3 shows the free-energy surfaces calculated for each umbrellas sampling simulation using the weighted histogram analysis method (WHAM) (72, 73). Dissociation constants calculated from each free-energy surface are shown in Table 1.

\section{Well-tempered metadynamics (WT-MetaD) simulations}

WT-MetaD simulations (74) were performed using the PLUMED 2.5 plugin (75-77) and the Martini model where chitin-protein interactions had been increased by $10 \%$. We used the distance $\left(r_{\text {chitin }}\right)$ and number of contacts (cut-off distance, $r_{0}=0.7 \mathrm{~nm}$ ) between aromatic residues in the putative substrate binding surfaces (CjCBM5: Y282, W283, Y296; CjCBM73: W371, Y378, 
W386) and the chitin surface in $15 \mu$ s long (15000 frames) simulations as collective variables to

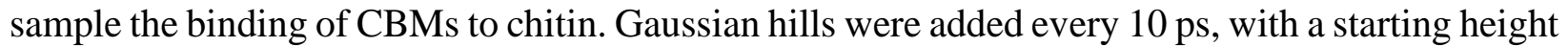
of $2.0 \mathrm{~kJ} \mathrm{~mol}^{-1}$, width of 0.5 , and bias factor of 50 . Figure $\mathrm{S} 4$ shows the evolution of the CVs and deposition of Gaussian hills over the course of the simulation.

Since the modeled chitin surface corresponds to the $\mathrm{x}, \mathrm{y}$-plane in the simulation box, $r_{\text {chitin }}$, was calculated using Equation 1 (shown below with $C j \mathrm{CBM} 5$ as an example), which uses the geometric center of the $\mathrm{z}$-coordinates, $z_{g c}$, calculated using Equation 2 . Here, $z_{i}$ is the $\mathrm{z}$-coordinate of each amino acid or chitin bead, and $N$ is the number of beads in the amino acid or chitin.

$$
\begin{gathered}
\mathrm{r}_{\text {chitin }}=\sqrt{\left(\mathrm{z}_{\mathrm{gc}, \mathrm{Y} 282}-\mathrm{z}_{\mathrm{gc}, \text { chitin }}\right)^{2}+\left(\mathrm{z}_{\mathrm{gc}, \mathrm{W} 283}-\mathrm{z}_{\mathrm{gc}, \text { chitin }}\right)^{2}+\left(\mathrm{z}_{\mathrm{gc}, \mathrm{Y} 296}-\mathrm{z}_{\mathrm{gc}, \mathrm{chitin}}\right)^{2}} \\
z_{g c}=\frac{\sum_{i}^{N} z_{i}}{N}
\end{gathered}
$$

The number of contacts between all beads in each amino acid and all beads in the chitin surface, $c_{i}$, were calculated using the COORDINATION routine in PLUMED 2.5 (i.e. Equation 3), with a cut-off distance $r_{0}=0.3 \mathrm{~nm}, r_{i}$ is the distance between all beads in each amino acid in the CBMs and all beads in the chitin surface.

$$
c_{i}=\frac{1-\left(r_{i} / r_{0}\right)^{6}}{1-\left(r_{i} / r_{0}\right)^{12}}
$$

Weights for each frame, $w_{i}$, were calculated from the bias in the WT-MetaD simulation using the REWEIGHT_BIAS routine in PLUMED 2.5 (see (78) and https://www.plumed.org/docv2.5/user-doc/html/_r_e_w_e_i_g_h_t_b_i_a_s.html for details). The weighted average number of contacts, $\langle c\rangle_{w}$, was calculated using Equation 4 and errors in $\langle c\rangle_{w}$ were estimated using block analysis (30).

$$
\langle c\rangle_{\mathrm{w}}=\sum \mathrm{w}_{\mathrm{i}} \cdot \mathrm{c}_{\mathrm{i}}
$$

\section{Dissociation constants from umbrella-sampling simulations}

A binding path for each CBM that included bound and unbound conformations was selected from the WT-MetaD simulations. Umbrella-sampling simulations were performed by running twentythree 100 ns-long replicas using the PLUMED 2.5 plugin, where the distance between the putative binding surface and the chitin surface $(r)$ was restrained from 0.0 to $4.4 \mathrm{~nm}$ in steps of $0.2 \mathrm{~nm}$ using a harmonic restraint with a force constant of $100 \mathrm{~kJ} \mathrm{~mol}^{-1} \mathrm{~nm}^{-1}$. Free-energy surfaces were calculated using the weighted-histogram analysis method (WHAM) $(72,73)$, where the errors were estimated by Monte-Carlo resampling. Dissociation constants $\left(K_{d}\right)$ were calculated from the freeenergy surfaces (Figure S3) by using Equations 5-7, where $r_{0}=0.0 \mathrm{~nm}$ (i.e. the minimum distance that for which WHAM calculated a non-zero probability), $r_{c}=1.85 \mathrm{~nm}, k_{B}$ is Boltzmann's constant, $\mathrm{T}$ is the temperature in Kelvin, $P_{0}$ is the protein concentration in the simulations, $\mathrm{N}_{\mathrm{A}}$ is 
Avogadro's number, $\mathrm{V}_{\mathrm{box}}$ is the volume of the simulation box, and $\dot{\alpha}=4.38 \mathrm{~nm}$ is the upper limit of $r_{\text {chitin. }}$

$$
\begin{gathered}
\mathrm{K}_{\mathrm{d}}=\mathrm{e}^{\frac{-\Delta \mathrm{G}_{\text {binding }}}{\mathrm{k}_{\mathrm{B}} \mathrm{T}}} \\
\Delta \mathrm{G}_{\text {binding }}=k_{B} T \ln \left[\frac{\int_{r_{c}}^{\dot{\alpha}} \exp \left(\frac{-P M F\left(r_{\text {chitin }}\right)}{k_{B} \mathrm{~T}}\right) \mathrm{d} r_{\text {chitin }}}{\int_{r_{0}}^{r_{c}} \exp \left(\frac{-P M F\left(r_{\text {chitin }}\right)}{k_{B} \mathrm{~T}}\right) \mathrm{d} r_{\text {chitin }}}\right]+k_{B} \mathrm{~T} \ln P_{0} \\
P_{0}=\frac{\left(1 / \mathrm{N}_{\mathrm{A}}\right)}{\mathrm{V}_{\mathrm{box}}}=\frac{\left(1 / 6.02 \cdot 10^{23} \mathrm{~mol}\right)}{16 \mathrm{~nm}^{3}} \approx 0.001 \mathrm{~mol} / \mathrm{L}
\end{gathered}
$$

All the data and PLUMED input files required to reproduce the simulation results reported in this article are available online at https://github.com/gcourtade/papers/tree/master/2021/CBM5CBM73-MetaD-US and on PLUMED-NEST (www.plumed-nest.org), the public repository of the PLUMED consortium (75) as plumID: 21.015.

\section{Acknowledgments}

This work was funded by The Novo Nordisk Foundation, project numbers NNF18OC0055736 (ZF) and NNF18OC0032242 (GC) and by the Research Council of Norway through projects 226244, 247001, 262853 \& 269408. Y.W. and K.L.-L. were supported by the BRAINSTRUC structural biology initiative from the Lundbeck Foundation.

\section{Conflict of interest}

The authors declare that they have no conflicts of interest.

\section{References}

1. Gooday, G. W. (1990) The Ecology of Chitin Degradation BT - Advances in Microbial Ecology (Marshall, K. C. ed), pp. 387-430, Springer US, Boston, MA, 10.1007/978-1-4684-7612-5_10

2. Igarashi, K., Uchihashi, T., Uchiyama, T., Sugimoto, H., Wada, M., Suzuki, K., Sakuda, S., Ando, T., Watanabe, T., and Samejima, M. (2014) Two-way traffic of glycoside hydrolase family 18 processive chitinases on crystalline chitin. Nat. Commun. 5, 3975

3. Hult, E., Katouno, F., Uchiyama, T., Watanabe, T., and Sugiyama, J. (2005) Molecular directionality in crystalline $\beta$-chitin: hydrolysis by chitinases A and B from Serratia marcescens 2170. Biochem. J. 388, 851-856

4. Vaaje-Kolstad, G., Horn, S. J., Sørlie, M., and Eijsink, V. G. H. (2013) The chitinolytic machinery of Serratia marcescens-a model system for enzymatic degradation of recalcitrant polysaccharides. FEBS J. 280, 3028-3049

5. Nakagawa, Y. S., Eijsink, V. G. H., Totani, K., and Vaaje-Kolstad, G. (2013) Conversion of $\alpha$ chitin substrates with varying particle size and crystallinity reveals substrate preferences of the chitinases and lytic polysaccharide monooxygenase of Serratia marcescens. J. Agric. Food Chem. 
61, 11061-11066

6. Vaaje-Kolstad, G., Westereng, B., Horn, S. J., Liu, Z., Zhai, H., Sørlie, M., and Eijsink, V. G. H. H. (2010) An oxidative enzyme boosting the enzymatic conversion of recalcitrant polysaccharides. Science. 330, 219-222

7. Vaaje-Kolstad, G., Horn, S. J., van Aalten, D. M. F. F., Synstad, B., and Eijsink, V. G. H. H. (2005) The non-catalytic chitin-binding protein CBP21 from Serratia marcescens is essential for chitin degradation. J. Biol. Chem. 280, 28492-28497

8. Quinlan, R. J., Sweeney, M. D., Lo Leggio, L., Otten, H., Poulsen, J.-C. N., Johansen, K. S., Krogh, K. B. R. M., Jørgensen, C. I., Tovborg, M., Anthonsen, A., Tryfona, T., Walter, C. P., Dupree, P., Xu, F., Davies, G. J., and Walton, P. H. (2011) Insights into the oxidative degradation of cellulose by a copper metalloenzyme that exploits biomass components. Proc. Natl. Acad. Sci. U. S. A. 108, 15079-15084

9. Forsberg, Z., Vaaje-Kolstad, G., Westereng, B., Bunæs, A. C., Stenstrøm, Y., MacKenzie, A., Sørlie, M., Horn, S. J., and Eijsink, V. G. H. (2011) Cleavage of cellulose by a CBM33 protein. Protein Sci. 20, 1479-1483

10. Eibinger, M., Ganner, T., Bubner, P., Rosker, S., Kracher, D., Haltrich, D., Ludwig, R., Plank, H., and Nidetzky, B. (2014) Cellulose surface degradation by a lytic polysaccharide monooxygenase and its effect on cellulase hydrolytic efficiency. J. Biol. Chem. 289, 35929-35938

11. Phillips, C. M., Beeson, W. T., Cate, J. H., and Marletta, M. A. (2011) Cellobiose dehydrogenase and a copper-dependent polysaccharide monooxygenase potentiate cellulose degradation by Neurospora crassa. ACS Chem. Biol. 6, 1399-1406

12. Agger, J. W., Isaksen, T., Várnai, A., Vidal-Melgosa, S., Willats, W. G. T., Ludwig, R., Horn, S. J., Eijsink, V. G. H., and Westereng, B. (2014) Discovery of LPMO activity on hemicelluloses shows the importance of oxidative processes in plant cell wall degradation. Proc. Natl. Acad. Sci. U. S. A. 111, 6287-6292

13. Vu, V. V, Beeson, W. T., Span, E. A., Farquhar, E. R., and Marletta, M. A. (2014) A family of starch-active polysaccharide monooxygenases. Proc. Natl. Acad. Sci. U. S. A. 111, 13822-13827

14. Vermaas, J. V., Crowley, M. F., Beckham, G. T., and Payne, C. M. (2015) Effects of lytic polysaccharide monooxygenase oxidation on cellulose structure and binding of oxidized cellulose oligomers to cellulases. J. Phys. Chem. B. 119, 6129-6143

15. Lombard, V., Golaconda Ramulu, H., Drula, E., Coutinho, P. M., and Henrissat, B. (2014) The carbohydrate-active enzymes database (CAZy) in 2013. Nucleic Acids Res. 42, 490-495

16. Boraston, A. B., Bolam, D. N., Gilbert, H. J., and Davies, G. J. (2004) Carbohydrate-binding modules: fine-tuning polysaccharide recognition. Biochem. J. 382, 769-781

17. Gilbert, H. J., Knox, J. P., and Boraston, A. B. (2013) Advances in understanding the molecular basis of plant cell wall polysaccharide recognition by carbohydrate-binding modules. Curr. Opin. Struct. Biol. 23, 669-677

18. Crouch, L. I., Labourel, A., Walton, P. H., Davies, G. J., and Gilbert, H. J. (2016) The contribution of non-catalytic carbohydrate binding modules to the activity lytic polysaccharide monooxygenases. J. Biol. Chem. 291, 7439-7449

19. Chalak, A., Villares, A., Moreau, C., Haon, M., Grisel, S., Orlando, A., Gimbert, I. H., Labourel, A., Cathala, B., and Berrin, J. G. (2019) Influence of the carbohydrate - binding module on the activity of a fungal AA9 lytic polysaccharide monooxygenase on cellulosic substrates. Biotechnol. Biofuels. 12, 206

20. Forsberg, Z., Nelson, C. E., Dalhus, B., Mekasha, S., Loose, J. S. M., Crouch, L. I., Røhr, Å. K., Gardner, J. G., Eijsink, V. G. H., and Vaaje-Kolstad, G. (2016) Structural and functional analysis of a lytic polysaccharide monooxygenase important for efficient utilization of chitin in Cellvibrio japonicus. J. Biol. Chem. 291, 7300-7312

21. Forsberg, Z., Bastien, B., Gullesen, J., Dalhus, B., Vaaje-Kolstad, G., Eijsink, V. G. H. H., Bissaro, B., Gullesen, J., Dalhus, B., Vaaje-Kolstad, G., and Eijsink, V. G. H. H. (2018) Structural determinants of bacterial lytic polysaccharide monooxygenase functionality. J. Biol. Chem. 293, 
$1397-1412$

22. Courtade, G., Forsberg, Z., Heggset, E. B., Eijsink, V. G. H., and Aachmann, F. L. (2018) The carbohydrate-binding module and linker of a modular lytic polysaccharide monooxygenase promote localized cellulose oxidation. J. Biol. Chem. 293, 13006-13015

23. Lehtiö, J., Sugiyama, J., Gustavsson, M., Fransson, L., Linder, M., and Teeri, T. T. (2003) The binding specificity and affinity determinants of family 1 and family 3 cellulose binding modules. Proc. Natl. Acad. Sci. U. S. A. 100, 484-489

24. Bayer, E. A., Chanzy, H., Lamed, R., and Shoham, Y. (1998) Cellulose, cellulases and cellulosomes. Curr. Opin. Struct. Biol. 8, 548-557

25. Brunecky, R., Alahuhta, M., Xu, Q., Donohoe, B. S., Crowley, M. F., Kataeva, I. A., Yang, S.-J., Resch, M. G., Adams, M. W. W., Lunin, V. V, Himmel, M. E., and Bomble, Y. J. (2013) Revealing nature's cellulase diversity: the digestion mechanism of Caldicellulosiruptor bescii CelA. Science. 342, 1513-1516

26. Bayer, E. A., Shoham, Y., and Lamed, R. (2006) Cellulose-decomposing Bacteria and Their Enzyme Systems. Prokaryotes. 2, 578-617

27. Brun, E., Moriaud, F., Gans, P., Blackledge, M. J., Barras, F., and Marion, D. (1997) Solution structure of the cellulose-binding domain of the endoglucanase $\mathrm{Z}$ secreted by Erwinia chrysanthemi. Biochemistry. 36, 16074-16086

28. Hudson, K. L., Bartlett, G. J., Diehl, R. C., Agirre, J., Gallagher, T., Kiessling, L. L., and Woolfson, D. N. (2015) Carbohydrate-aromatic interactions in proteins. J. Am. Chem. Soc. 137, $15152-15160$

29. Monticelli, L., Kandasamy, S. K., Periole, X., Larson, R. G., Tieleman, D. P., and Marrink, S. J. (2008) The MARTINI coarse-grained force field: Extension to proteins. J. Chem. Theory Comput. 4, 819-834

30. Flyvbjerg, H., and Petersen, H. G. (1989) Error estimates on averages of correlated data. J. Chem. Phys. 91, 461-466

31. Mutahir, Z., Mekasha, S., Loose, J. S. M., Abbas, F., Vaaje-Kolstad, G., Eijsink, V. G. H., and Forsberg, Z. (2018) Characterization and synergistic action of a tetra-modular lytic polysaccharide monooxygenase from Bacillus cereus. FEBS Lett. 592, 2562-2571

32. Ikegami, T., Okada, T., Hashimoto, M., Seino, S., Watanabe, T., and Shirakawa, M. (2000) Solution structure of the chitin-binding domain of Bacillus circulans WL-12 chitinase A1. J. Biol. Chem. 275, 13654-13661

33. Akagi, K. I., Watanabe, J., Hara, M., Kezuka, Y., Chikaishi, E., Yamaguchi, T., Akutsu, H., Nonaka, T., Watanabe, T., and Ikegami, T. (2006) Identification of the substrate interaction region of the chitin-binding domain of Streptomyces griseus chitinase C. J. Biochem. 139, 483-493

34. Mine, S., Nakamura, T., Sato, T., Ikegami, T., and Uegaki, K. (2014) Solution structure of the chitin-binding domain 1 (ChBD1) of a hyperthermophilic chitinase from Pyrococcus furiosus. $J$. Biochem. 155, 115-122

35. Simpson, H. D., and Barras, F. (1999) Functional analysis of the carbohydrate-binding domains of Erwinia chrysanthemi Cel5 (endoglucanase Z) and an Escherichia coli putative chitinase. J. Bacteriol. 181, 4611-4616

36. van Aalten, D. M., Synstad, B., Brurberg, M. B., Hough, E., Riise, B. W., Eijsink, V. G., and Wierenga, R. K. (2000) Structure of a two-domain chitotriosidase from Serratia marcescens at 1.9-A resolution. Proc. Natl. Acad. Sci. U. S. A. 97, 5842-5847

37. Malecki, P. H., Raczynska, J. E., Vorgias, C. E., and Rypniewski, W. (2013) Structure of a complete four-domain chitinase from Moritella marina, a marine psychrophilic bacterium. Acta Crystallogr. Sect. D Biol. Crystallogr. 69, 821-829

38. Hara, M., Sugimoto, H., Uemura, M., Akagi, K., Suzuki, K., Ikegami, T., and Watanabe, T. (2013) Involvement of Gln679, in addition to Trp687, in chitin-binding activity of the chitin-binding domain of chitinase A1 from Bacillus circulans WL-12. J. Biochem. 154, 185-193

39. Mattinen, M. L., Kontteli, M., Kerovuo, J., Linder, M., Annila, A., Lindeberg, G., Reinikainen, T., 
and Drakenberg, T. (1997) Three-dimensional structures of three engineered cellulose-binding domains of cellobiohydrolase I from Trichoderma reesei. Protein Sci. 6, 294-303

40. Tormo, J., Lamed, R., Chirino, A. J., Morag, E., Bayer, E. A., Shoham, Y., and Steitz, T. A. (1996) Crystal structure of a bacterial family-III cellulose-binding domain: a general mechanism for attachment to cellulose. EMBO J. 15, 5739-5751

41. Xu, G. Y., Ong, E., Gilkes, N. R., Kilburn, D. G., Muhandiram, D. R., Harris-Brandts, M., Carver, J. P., Kay, L. E., and Harvey, T. S. (1995) Solution structure of a cellulose-binding domain from Cellulomonas fimi by nuclear magnetic resonance spectroscopy. Biochemistry. 34, 6993-7009

42. Nagy, T., Simpson, P., Williamson, M. P., Hazlewood, G. P., Gilbert, H. J., and Orosz, L. (1998) All three surface tryptophans in Type IIa cellulose binding domains play a pivotal role in binding both soluble and insoluble ligands. FEBS Lett. 429, 312-316

43. Bissaro, B., Røhr, Å. K., Müller, G., Chylenski, P., Skaugen, M., Forsberg, Z., Horn, S. J., VaajeKolstad, G., and Eijsink, V. G. H. (2017) Oxidative cleavage of polysaccharides by monocopper enzymes depends on $\mathrm{H}_{2} \mathrm{O}_{2}$. Nat. Chem. Biol. 13, 1123-1128

44. Mekasha, S., Tuveng, T. R., Askarian, F., Choudhary, S., Schmidt-Dannert, C., Niebisch, A., Modregger, J., Vaaje-Kolstad, G., and Eijsink, V. G. H. (2020) A tri-modular bacterial enzyme combining hydrolytic activity with oxidative glycosidic bond cleavage efficiently degrades chitin. J. Biol. Chem. 295, 9134-9146

45. Manoil, C., and Beckwith, J. (1986) A genetic approach to analyzing membrane protein topology. Science. 233, 1403-1408

46. Brurberg, M. B., Eijsink, V. G. H., and Nes, I. F. (1994) Characterization of a chitinase gene (chiA) from Serratia marcescens BJL200 and one-step purification of the gene product. FEMS Microbiol. Lett. 124, 399-404

47. Loose, J. S. M., Forsberg, Z., Fraaije, M. W., Eijsink, V. G. H., and Vaaje-Kolstad, G. (2014) A rapid quantitative activity assay shows that the Vibrio cholerae colonization factor GbpA is an active lytic polysaccharide monooxygenase. FEBS Lett. 588, 3435-3440

48. Heuts, D. P. H. M., Winter, R. T., Damsma, G. E., Janssen, D. B., and Fraaije, M. W. (2008) The role of double covalent flavin binding in chito-oligosaccharide oxidase from Fusarium graminearum. Biochem. J. 413, 175-183

49. Huynh, K., and Partch, C. L. (2015) Analysis of protein stability and ligand interactions by thermal shift assay. Curr. Protoc. Protein Sci. 79, 28.9.1-28.9.14

50. Zhang, H., Neal, S., and Wishart, D. S. (2003) RefDB: A database of uniformly referenced protein chemical shifts. J. Biomol. NMR. 25, 173-95

51. Lescop, E., Schanda, P., and Brutscher, B. (2007) A set of BEST triple-resonance experiments for time-optimized protein resonance assignment. J. Magn. Reson. 187, 163-169

52. Keller, R. (2004) The Computer Aided Resonance Assignment Tutorial, CANTINA Verlag, Goldau, Switzerland

53. Shen, Y., and Bax, A. (2013) Protein backbone and sidechain torsion angles predicted from NMR chemical shifts using artificial neural networks. J. Biomol. NMR. 56, 227-241

54. Güntert, P. (2004) Automated NMR Structure Calculation With CYANA. Methods Mol. Biol. 278, 353-378

55. Krieger, E., Koraimann, G., and Vriend, G. (2002) Increasing the precision of comparative models with YASARA NOVA - a self-parameterizing force field. Proteins Struct. Funct. Bioinforma. 47, 393-402

56. Essmann, U., Perera, L., Berkowitz, M. L., Darden, T., Lee, H., and Pedersen, L. G. (1995) A smooth particle mesh Ewald method. J. Chem. Phys. 103, 8577-8593

57. Krieger, E., Joo, K., Lee, J., Lee, J., Raman, S., Thompson, J., Tyka, M., Baker, D., and Karplus, K. (2009) Improving physical realism, stereochemistry, and side-chain accuracy in homology modeling: Four approaches that performed well in CASP8. Proteins Struct. Funct. Bioinforma. 77, $114-122$

58. Shindyalov, I. N., and Bourne, P. E. (1998) Protein structure alignment by incremental 
combinatorial extension (CE) of the optimal path. Protein Eng. 11, 739-747

59. Mulder, F. A. A., Schipper, D., Bott, R., and Boelens, R. (1999) Altered flexibility in the substrate-binding site of related native and engineered high-alkaline Bacillus subtilisins. J. Mol. Biol. 292, 111-123

60. Sikorski, P., Hori, R., and Wada, M. (2009) Revisit of $\alpha$-chitin crystal structure using high resolution X-ray diffraction data. Biomacromolecules. 10, 1100-1105

61. Pettersen, E. F., Goddard, T. D., Huang, C. C., Couch, G. S., Greenblatt, D. M., Meng, E. C., and Ferrin, T. E. (2004) UCSF Chimera-A visualization system for exploratory research and analysis. J. Comput. Chem. 25, 1605-1612

62. Yu, Z., and Lau, D. (2015) Development of a coarse-grained $\alpha$-chitin model on the basis of MARTINI forcefield. J. Mol. Model. 21, 128

63. Abraham, M. J., Murtola, T., Schulz, R., Páll, S., Smith, J. C., Hess, B., and Lindahl, E. (2015) GROMACS: High performance molecular simulations through multi-level parallelism from laptops to supercomputers. SoftwareX. 1-2, 19-25

64. de Jong, D. H., Singh, G., Bennett, W. F. D., Arnarez, C., Wassenaar, T. A., Schäfer, L. V, Periole, X., Tieleman, D. P., and Marrink, S. J. (2013) Improved parameters for the Martini coarse-grained protein force field. J. Chem. Theory Comput. 9, 687-697

65. Periole, X., Cavalli, M., Marrink, S.-J., and Ceruso, M. A. (2009) Combining an elastic network with a coarse-grained molecular force field: structure, dynamics, and intermolecular recognition. $J$. Chem. Theory Comput. 5, 2531-2543

66. DeLano, W. L., and Lam, J. (2005) PyMOL: A communications tool for computational models. Abstr. Pap. Am. Chem. Soc. 230, 1371-1372

67. Bussi, G., Donadio, D., and Parrinello, M. (2007) Canonical sampling through velocity rescaling. J. Chem. Phys. 126, 014101

68. Parrinello, M., and Rahman, A. (1981) Polymorphic transitions in single crystals: A new molecular dynamics method. J. Appl. Phys. 52, 7182-7190

69. Páll, S., and Hess, B. (2013) A flexible algorithm for calculating pair interactions on SIMD architectures. Comput. Phys. Commun. 184, 2641-2650

70. Vickery, O. N., and Stansfeld, P. J. (2021) CG2AT2: An Enhanced Fragment-based approach for Serial Multi-scale Molecular Dynamics simulations. bioRxiv. 10.1101/2021.03.25.437005

71. Larsen, A. H., Wang, Y., Bottaro, S., Grudinin, S., Arleth, L., and Lindorff-Larsen, K. (2020) Combining molecular dynamics simulations with small-angle X-ray and neutron scattering data to study multi-domain proteins in solution. PLoS Comput. Biol. 16, e1007870

72. Grossfield, A. (2020) WHAM: the weighted histogram analysis method, version 2.0.9

73. Kumar, S., Rosenberg, J. M., Bouzida, D., Swendsen, R. H., and Kollman, P. A. (1992) The weighted histogram analysis method for free-energy calculations on biomolecules. I. The method. J. Comput. Chem. 13, 1011-1021

74. Barducci, A., Bussi, G., and Parrinello, M. (2008) Well-tempered metadynamics: a smoothly converging and tunable free-energy method. Phys. Rev. Lett. 100, 020603

75. The PLUMED Consortium (2019) Promoting transparency and reproducibility in enhanced molecular simulations. Nat. Methods. 16, 670-673

76. Tribello, G. A., Bonomi, M., Branduardi, D., Camilloni, C., and Bussi, G. (2014) PLUMED 2: New feathers for an old bird. Comput. Phys. Commun. 185, 604-613

77. Bonomi, M., Branduardi, D., Bussi, G., Camilloni, C., Provasi, D., Raiteri, P., Donadio, D., Marinelli, F., Pietrucci, F., Broglia, R. A., and Parrinello, M. (2009) PLUMED: A portable plugin for free-energy calculations with molecular dynamics. Comput. Phys. Commun. 180, 1961-1972

78. Tiwary, P., and Parrinello, M. (2015) A time-independent free energy estimator for metadynamics. J. Phys. Chem. B. 119, 736-742 